\title{
EQUIVARIANT DISCRETIZATIONS OF DIFFUSIONS, RANDOM WALKS, AND HARMONIC FUNCTIONS
}

\author{
WERNER BALLMANN AND PANAGIOTIS POLYMERAKIS
}

\begin{abstract}
For covering spaces and properly discontinuous actions with compatible diffusion processes, we discuss Lyons-Sullivan discretizations of the processes and the associated function theory.
\end{abstract}

\section{CONTENTS}

1. Introduction

2. Preliminaries

3. Lyons-Sullivan discretization

4. Diffusion processes and covering projections

5. Diffusion processes and properly discontinuous actions

6. Random walks and harmonic functions

References

\section{INTRODUCTION}

We are interested in spaces of bounded or positive $\lambda$-harmonic functions on Riemannian manifolds and, more generally, spaces of bounded or positive harmonic functions of diffusion operators. Our work is motivated by the articles [Su of Sullivan and $\mathrm{LS}$ of Lyons and Sullivan.

To a sufficiently large discrete subset $X$ in a connected Riemannian manifold $M$, Lyons and Sullivan associate a family of probability measures $\mu=\left(\mu_{y}\right)_{y \in M}$ on $X$ which has a number of important properties. Among others, Lyons and Sullivan show that

$$
h(y)=\mu_{y}(h):=\sum_{x \in X} \mu_{y}(x) h(x)
$$

for all bounded harmonic functions $h$ on $M$ and $y \in M$. Furthermore, the support of each of the measures $\mu_{y}$ is all of $X$. We call $\mu$ the family of Lyons-Sullivan measures or, as a shorthand, LS-measures. They depend on the choice of data, which we call $L S$-data, and these exist if $X$ is *-recurrent in the terminology of Lyons and Sullivan. We use the same concept, but in the context of diffusion operators and in the form used in [BL2] and develop and extend results from [LS], $\mathrm{K} 2$, [LZ, and BL2] in different directions.

Date: June 28, 2019.

2010 Mathematics Subject Classification. 53C99, 58J65, 60G50.

Key words and phrases. Diffusion operator, random walk, Martin boundary, Poisson boundary, harmonic function, covering projection, properly discontinuous action, discretization.

We would like to thank François Ledrappier, Henrik Matthiesen, and Mikhail Zaidenberg for helpful comments. We would also like to thank the Max Planck Institute for Mathematics and the Hausdorff Center for Mathematics in Bonn for their support and hospitality. 
Let $M$ be a connected manifold and $L$ be an elliptic diffusion operator on $M$ that is symmetric on $C_{c}^{\infty}(M)$ with respect to a smooth volume element (see Section 2.1). We say that a smooth function $h$ on $M$ is $L$-harmonic if $L h=0$.

Let $\left(D_{t}\right)_{t \geq 0}$ be the diffusion process on $M$ with generator $L$ (see Section 2.1). We say that $M$ is $L$-complete, $L$-recurrent, or $L$-transient if $\left(D_{t}\right)$ is stochastically complete, recurrent, or transient, respectively. Note that $M$ is either $L$-recurrent or $L$-transient and that $L$-recurrence implies $L$-completeness.

Example 1.2. If $M$ is compact, then $M$ is $L$-recurrent.

In what follows, we state our main results in the case of a covering $\pi: M \rightarrow N$, where the operator $L$ on $M$ is the pull back of a diffusion operator $L_{0}$ on $N$. We have similar results in the case of a countable group acting properly discontinuously and $L$-equivariantly on $M$. In the literature, the case of normal Riemannian coverings (with $L, L_{0}$ the Laplacian) is usually considered. In our final section, we also discuss related results for random walks, that is, Markov chains on countable sets.

We fix a fiber $X$ of $\pi$. Then $X$ is $*$-recurrent in the sense of Lyons and Sullivan exactly in the case where $N$ is $L_{0}$-recurrent, and the latter is assumed throughout the following. By Example 1.2, the assumption is satisfied whenever the base manifold $N$ is compact.

We let $\mu=\left(\mu_{y}\right)_{y \in M}$ be the $L S$-probability measures on $X$ associated to a choice of $L S$-data for $X$. We say that a function $h$ on $X$ is $\mu$-harmonic if it satisfies (1.1) for all $y \in X$.

1.1. Main results in the cocompact case. We let $\mathcal{H}^{+}(M, L)$ and $\mathcal{H}^{+}(X, \mu)$ be the cones of positive $L$-harmonic functions on $M$ and positive $\mu$-harmonic functions on $X$, respectively. Constant functions on $M$ and $X$ are $L$-harmonic and $\mu$-harmonic, respectively. If $M$ is $L$-recurrent, then any positive $L$-harmonic function on $M$ is constant. Similarly, if the $\mu$-random walk on $X$ is recurrent, then any positive $\mu$-harmonic function on $X$ is constant.

Theorem A. Suppose that $N$ is compact and that $X$ is endowed with the family $\mu$ of LS-measures associated to appropriate LS-data. Then

(1) for any $h \in \mathcal{H}^{+}(M, L)$, the restriction $\left.h\right|_{X}$ of $h$ to $X$ belongs to $\mathcal{H}^{+}(X, \mu)$. More precisely, $h(y)=\mu_{y}(h)$ for all $y \in M$;

(2) the restriction map

$$
\mathcal{H}^{+}(M, L) \rightarrow \mathcal{H}^{+}(X, \mu),\left.\quad h \mapsto h\right|_{X},
$$

is a $\Gamma$-equivariant isomorphism of cones.

The meaning of the attribute 'appropriate' (here and below) will be specified in Section 4. We show in Lemma 4.4 that appropriate $L S$-data always exist.

In the case of the Laplacian and with a different choice of $L S$-data, Theorem $\mathrm{A} 1$ is due to Lyons and Sullivan $[\mathrm{LS}$, Theorem 6]. Theorem $\mathrm{A} 2 \mathrm{2}$ is new even for the Laplacian. Using that our choice of $L S$-data leads to symmetric random walks on $X$ and that positive harmonic functions of irreducible symmetric random walks on nilpotent groups are constant, Theorem A implies [LS, Theorem 1] which asserts, in the case where $\pi$ is a normal Riemannian covering with compact base, that any positive harmonic function on $M$ is constant if the group of covering transformations of $\pi$ is nilpotent.

In the case where $M$ is $L$-transient, the Martin boundary $\partial_{L} M$ and Martin compactification $\mathcal{M}(M, L)=M \cup \partial_{L} M$ of $M$ are defined (see Section 2.2 below). Similarly, if the $\mu$-random walk on $X$ is transient, we have the Martin boundary $\partial_{\mu} X$ and Martin compactification $\mathcal{M}(X, \mu)=X \cup \partial_{\mu} X$ of $X$. The minimal parts 
$\partial_{L}^{\min } M$ and $\partial_{L}^{\min }(X, \mu)$ of the Martin boundaries consist of minimal positive $L$ harmonic and minimal positive $\mu$-harmonic functions, respectively. The following result is new even in the case of the Laplacian.

Theorem B. Suppose that $N$ is compact, that $M$ is L-transient, and that $X$ is endowed with the family $\mu$ of $L S$-measures associated to appropriate $L S$-data. Then

(1) the $\mu$-random walk on $X$ is transient and the inclusion $X \rightarrow M$ extends to a $\Gamma$-equivariant homeomorphism

$$
\partial_{L}^{\min }(X, \mu) \rightarrow \partial_{L}^{\min }(M, L)
$$

(2) $\partial_{L}^{\min }(M, L)=\partial_{L} M$ if and only if $\partial_{L}^{\min }(X, \mu)=\partial_{\mu} X$.

1.2. Main results in the recurrent case. In contrast to the cocompact case, our arguments and results in this subsection are mostly straightforward extensions of corresponding arguments and results in the literature, where the case of the Laplacian and normal Riemannian coverings is usually considered. Nevertheless, there are differences and twists in the setup and argumentation which may make our exposition more accessible.

We denote by $\mathcal{H}^{\infty}(M, L)$ and $\mathcal{H}^{\infty}(X, \mu)$ the spaces of bounded $L$-harmonic functions on $M$ and bounded $\mu$-harmonic functions on $X$, respectively. Note that $\mathcal{H}^{\infty}(M, L)$ vanishes whenever $M$ is $L$-recurrent and that $\mathcal{H}^{\infty}(X, \mu)$ vanishes whenever the $\mu$-random walk on $X$ is recurrent.

Theorem C. Suppose that $N$ is $L_{0}$-recurrent and that $X$ is endowed with the family $\mu$ of $L S$-measures associated to appropriate $L S$-data. Then

(1) for any $h \in \mathcal{H}^{\infty}(M, L)$, the restriction $\left.h\right|_{X}$ of $h$ to $X$ belongs to $\mathcal{H}^{\infty}(X, \mu)$;

(2) for any $h \in \mathcal{H}^{+}(M, L)$, either $h$ is $\mu$-harmonic or strictly $\mu$-superharmonic on $X$. More precisely, either $h(y)=\mu_{y}(h)$ for all $y \in M$ or $h(y)>\mu_{y}(h)$ for all $y \in M$.

(3) the restriction map

$$
\mathcal{H}^{\infty}(M, L) \rightarrow \mathcal{H}^{\infty}(X, \mu),\left.\quad h \mapsto h\right|_{X},
$$

is a $\Gamma$-equivariant isomorphism of vector spaces.

(4) $M$ is L-transient if and only if the $\mu$-random walk on $X$ is transient.

In the case of the Laplacian, (11) is part of [LS, Theorem 5], (2) is [BL2, Theorem 1.10], (3) follows from [BL2, Theorem 1.11] and (with other assumptions on the $L S$-data) from [K2, Theorem 1], and (41) is part of [BL2, Theorem 2.7]. In our arguments, we follow BL2.

Lyons and Sullivan also discretize random Brownian paths in $M$ to $\mu$-random sequences in $X$, see [LS, Section 8]. In the proof of [K2, Theorem 1], their procedure was analyzed by Kaimanovich and applied (in [K2, Remark 1]) to Poisson boundaries (see Section 2.3 below).

Theorem D. Suppose that $N$ is $L_{0}$-recurrent and and that $X$ is endowed with the family $\mu$ of $L S$-measures associated to appropriate LS-data. Then

(1) LS-path discretization induces a $\Gamma$-equivariant isomorphism

$$
\mathcal{P}(M, L) \rightarrow \mathcal{P}(X, \mu)
$$

of Poisson boundaries.

(2) the inclusion $X \rightarrow M$ extends to a $\Gamma$-equivariant convex homeomorphism

$$
\mathcal{M}(X, \mu) \rightarrow \mathcal{M}(M, L) \cap \bar{X},
$$

where $\bar{X}$ denotes the closure of $X$ in $\mathcal{M}(M, L)$.

In the case of the Laplacian, (11) follows from [K2, Theorem 1 and Remark 1] and (2) from [BL2, Theorem 2.8]. In our proofs, we follow arguments in [LS, K2, BL2]. 
1.3. Main results for normal coverings. The following is an extension of LS, Theorem, p. 307], where the case of the Laplacian is considered. Our proof is considerably shorter than the one in [LS], but we pay for this by using $L S$-discretization.

Theorem E. Suppose that $\pi$ is normal and that $N$ is $L_{0}$-recurrent. Let $h$ be a minimal positive L-harmonic function on $M$. Then either $h$ is constant or there is a $\gamma \in \Gamma$ such that $\gamma^{*} h / h$ is unbounded.

Using their discretization of Brownian motion, Lyons and Sullivan showed that, in the case of a normal Riemannian covering $\pi: M \rightarrow N$, any bounded harmonic function on $M$ is constant if $N$ is recurrent and $\Gamma$ is $\omega$-hypercentral LS, Theorem $2]$ (where $\omega$ denotes the first infinite ordinal). This was generalized by Lin and Zaidenberg to $F C$-hypercentral groups LZ, Corollary 2.6] (see Section 2.4). More generally, they showed that bounded harmonic functions on $M$ are invariant under the $F C$-hypercenter of $\Gamma$ if $N$ is recurrent [LZ, Corollary 2.5].

Theorem F. If $\pi$ is normal and $N$ is $L_{0}$-recurrent, then any bounded L-harmonic function on $M$ is invariant under the $F C$-hypercenter of $\Gamma$. In particular, if $\Gamma$ is $F C$-hypercentral, then any bounded L-harmonic function on $M$ is constant.

Whereas Li and Zaidenberg use the Stone-Čech compactification of $\Gamma$ in their proof, we rely on $L S$-discretization. Nevertheless, our argumentation here draws heavily from [LZ and the earlier [Li].

The following result corresponds to [LS, Theorem 3'], except that the assumption on recurrence is not needed there (and used here for convenience only).

Theorem G. If $\pi$ is normal and $N$ is $L_{0}$-recurrent, then there is a $\Gamma$-invariant bounded projection $L^{\infty}(M) \rightarrow \mathcal{H}^{\infty}(M, L)$. In particular, if all bounded L-harmonic functions on $M$ are constant, then $\Gamma$ is amenable.

It seems that the proof in [LS] extends to the situation considered here. However, we remain in our setup and invoke $L S$-discretization.

1.4. The motivating example (after Sullivan $[\mathrm{Su}]$ ). Let $M$ be a connected Riemannian manifold and set

$$
\lambda_{0}=\lambda_{0}(M)=\inf R(f),
$$

where $f$ runs through all non-vanishing smooth functions on $M$ with compact support and $R(f)$ denotes the Rayleigh quotient of $f$. Recall that $\lambda_{0}$ is equal to the bottom of the spectrum of the Friedrichs extension of the (geometer's) Laplacian $\Delta$ of $M$, considered as an unbounded symmetric operator on the space $L^{2}(M)$ of square-integrable functions on $M$ with domain $C_{c}^{\infty}(M)$, the space of smooth functions on $M$ with compact support.

It is well known that $\lambda_{0}$ is the supremum over all $\lambda \in \mathbb{R}$ such that there is a positive $\lambda$-harmonic function $f: M \rightarrow \mathbb{R}$ (see, e.g., [CY, Theorem 7], [FS, Theorem 1], or $[\mathrm{Su}$, Theorem 2.1]). It is crucial that these $\lambda$-harmonic functions are not required to be square-integrable. In fact, by the above, $\lambda_{0}$ is exactly the border between positive and $L^{2}$-spectrum of $M$.

Remark 1.4. If the right action of $\pi_{1}(N, q)$ on $X=\pi^{-1}(q)$ is amenable, then $\lambda_{0}(M)=\lambda_{0}(N)$; see BMP. If the right action of $\pi_{1}(N, q)$ on $X$ is not amenable and $\lambda_{0}(N)$ does not belong to the essential spectrum of $N$, then $\lambda_{0}(M)>\lambda_{0}(N)$; see [P1].

Let $p=p(t, x, y)$ be the kernel of the minimal heat semigroup on $M$. Recall that $p(t, x, y)>0$ for all $t>0$ and $x, y \in M$. Say that $\lambda \in \mathbb{R}$ belongs to the Green's 
region of $M$ if

$$
\int_{0}^{\infty} e^{\lambda t} p(t, x, y) d t<\infty
$$

for some-and then any-pair of points $x \neq y$ in $M$. By $[\mathrm{Su}$, Theorem 2.6], the Green's region of $M$ is either $\left(-\infty, \lambda_{0}\right)$ or $\left(-\infty, \lambda_{0}\right]$. In the first case, $M$ is said to be $\lambda_{0}$ recurrent, in the second $\lambda_{0}$-transient. By [Su, Theorem 2.7], if $M$ is $\lambda_{0}$-recurrent, then positive $\lambda_{0}$-harmonic functions are constant multiples of one another. By $\mathrm{Su}$, Theorem 2.8], if $M$ has a square integrable $\lambda_{0}$-harmonic function, then the space of square integrable $\lambda_{0}$-harmonic functions on $M$ is generated by a square integrable positive $\lambda_{0}$-harmonic function and $M$ is $\lambda_{0}$-recurrent. In the $\lambda_{0}$-recurrent case, the associated $\lambda_{0}$-random motion with transition densities $e^{\lambda_{0} t} p(t, x, y) \varphi(y) / \varphi(x)$ with respect to the Riemannian volume element of $M$, where $\varphi$ is a positive $\lambda_{0}$-harmonic function on $M$, does not depend on the choice of $\varphi$ (by what was said above) and is recurrent, by [Su, Theorem 2.10]).

In the case $\lambda_{0}=0$, we also speak of harmonic functions and of recurrent or transient manifolds. In this case, the associated random walk is standard Brownian motion on $M$.

Example 1.6. If $M$ is compact or, more generally, complete and of finite volume, then $\lambda_{0}=0$, and constant functions are $\lambda_{0}$-harmonic and square-integrable.

Example 1.7. (Sullivan [Su, Theorem 2.21].) Let $M=\Gamma \backslash H^{m}$ be a complete hyperbolic manifold and suppose that $M$ is geometrically finite, that is, the action of $\Gamma$ on $H^{m}$ admits a fundamental domain with finitely many and totally geodesic sides. Then

$$
\lambda_{0}= \begin{cases}(m-1)^{2} / 4 & \text { if } d \leq(m-1) / 2, \\ d(m-1-d) & \text { if } d \geq(m-1) / 2,\end{cases}
$$

where $d=d(\Gamma)$ denotes the Hausdorff dimension of the limit set of $\Gamma$. If $d \geq$ $(m-1) / 2$, then $M$ is $\lambda_{0}$-recurrent. If $d>(m-1) / 2$, then $M$ has square-integrable positive $\lambda_{0}$-harmonic functions. (See also [Su, Theorem 2.17 and Corollary 2.18].)

Let $\pi: M \rightarrow N$ be a Riemannian covering of connected Riemannian manifolds with group $\Gamma$ of covering transformations and $X$ be the fiber of $\pi$ over some chosen point in $N$. Let $\lambda_{0}=\lambda_{0}(N)$, and assume that $N$ is $\lambda_{0}$-recurrent. Let $\varphi$ be the lift of a positive $\lambda_{0}$-harmonic function from $N$ to $M$ and dv be the Riemannian volume element of $M$. Then multiplication $m_{\varphi}$ by $\varphi$ gives a unitary transformation $L^{2}\left(M, \varphi^{2} \mathrm{dv}\right) \rightarrow \mathrm{L}^{2}(\mathrm{M}, \mathrm{dv})$ which intertwines the diffusion operator $L=L f=-\Delta f+2\langle\nabla \ln \varphi, \nabla f\rangle$ with the operator $-\Delta+\lambda_{0}$; see the computation in the beginning of [Su, Section 8] (compare also with Section 2.1 below). The associated diffusion process has transition densities $e^{\lambda_{0} t} p(t, x, y) / \varphi(x) \varphi(y)$ with respect to the volume element $\varphi^{2} \mathrm{dv}$. Therefore, since $N$ is $\lambda_{0}$-recurrent, $X$ is $*$-recurrent in the sense of Lyons and Sullivan. Hence the above results apply if $N$ is compact (then $\left.\lambda_{0}=0\right)$ or $\lambda_{0}$-recurrent, respectively.

1.5. Discussion. An important consequence of our results is that, under specific conditions, problems about $L$-harmonic functions on $M$, minimal Martin boundaries $\partial_{L}^{\min } M$, and Poisson boundaries $\mathcal{P}(M, L)$ are equivalent to problems about $\mu$-harmonic functions on $X$, minimal Martin boundaries $\partial_{\mu}^{\min } X$, and Poisson boundaries $\mathcal{P}(X, \mu)$. The most basic problems in this direction are the triviality of the Poisson and Martin boundaries, that is, the property that $M$ does not carry nonconstant bounded or positive $L$-harmonic functions, and correspondingly for random walks. These properties are referred to as the Liouville and strong Liouville property, respectively. 
Let $\pi: M \rightarrow N$ be a normal Riemannian covering of connected manifolds with group $\Gamma$ of covering transformations. Lyons and Sullivan showed that $\Gamma$ is amenable if $M$ has the Liouville property, that is, if $\mathcal{P}(M, \Delta)$ is trivial. The converse does not hold. In fact, Lyons and Sullivan gave examples of Riemannian covers $M$ of closed surfaces $N$, where $\Gamma$ is two-step solvable and $\mathcal{P}(M, \Delta)$ is nontrivial [LS, pp. 299-300]. In the same direction, Erschler showed that $\mathcal{P}(M, \Delta)$ is nontrivial if $N$ is closed and $\Gamma$ is a Baumslag group $B_{d}$ with $d \geq 3$ [Er, Theorem 5.2], a two-step solvable and finitely presentable group. In her proof, Erschler uses the isomorphism $\mathcal{P}(M, \Delta) \cong \mathcal{P}(X, \mu)$, where $X$ is a fibre of $\pi$ and $\mu$ an associated family of LyonsSullivan measures on $X$. Note also that, since $B_{d}$ is finitely presentable, $N$ can be chosen to have fundamental group $\Gamma=B_{d}$ and $M$ to be the universal covering space of $N$.

At this point, it is not clear whether there are any non-trivial conditions on $\Gamma$ which are equivalent to the Liouville property of $M$ if $N$ is closed or recurrent. As for sufficient conditions, Lyons and Sullivan proved that $\mathcal{P}(M, \Delta)$ is trivial in the case where $N$ is recurrent and $\Gamma$ is $\omega$-hypercentral [LS, Theorem 2]. This was extended by Lin and Zaidenberg to the case where $\Gamma$ is $F C$-hypercentral [LZ, Corollary 2.6]. Kaimanovich showed by analytic methods that $\mathcal{P}(M, \Delta)$ is trivial if $N$ is closed and $\Gamma$ is polycyclic or of subexponential growth [K1, Theorem 8 and Corollary 1]. All the above results can also be shown for elliptic diffusion operators by using the isomorphisms $\mathcal{H}^{\infty}(M, L) \cong \mathcal{H}^{\infty}(X, \mu)$ and between $\mathcal{P}(M, \Delta) \cong \mathcal{P}(X, \mu)$, respectively. In the present article, (the second part of) Theorem $\mathrm{F}$ is an example where this strategy applies. Another one is the consequence of Theorem $\mathrm{G}$ that $M$ admits positive $\lambda_{0}(N)$-harmonic functions which are not $\Gamma$-invariant, provided that $N$ is $\lambda_{0}$-recurrent and $\Gamma$ is not amenable. In the context of the Liouville property, the entropy criterion of Kaimanovich and Vershik for random walks on groups is also of interest [KV, Theorem 1.1].

The strong Liouville property, that is, the triviality of $\partial_{\Delta}^{\min } M$, was obtained by a direct argument by Lyons and Sullivan in the case where $N$ is closed and $\Gamma$ is nilpotent [LS, Theorem 1]. As we mentioned further up, the extension of this result to elliptic diffusion operators is also an immediate consequence of the isomorphism $\mathcal{H}^{+}(M, L) \cong \mathcal{H}^{+}(X, \mu)$ and the corresponding result for nilpotent groups (see $\mathrm{CD}, \mathrm{Ma}$ and also Corollary 6.16). Lyons and Sullivan point out the example of a rank two Abelian covering space of the two-sphere with four points removed which admits non-constant positive harmonic functions, thus showing that the assumption that $N$ is closed is essential. This example was generalized and systematized by Epstein to Abelian covers of surfaces of finite type Ep, Theorem $3]$.

For the case where $N$ is closed, Lyons and Sullivan posed the problem whether $\Gamma$ has subexponential growth if and only if $M$ has the strong Liouville property [LS, p. 305]. Bougerol and Elie obtained the converse direction of this under the assumption that $\Gamma$ is linear [BE, Theorem 1.6] and also [BBE, Theorem 1.1]. Recently, the second author of this article proved the converse direction in full generality $[\mathrm{P} 2$.

By the well known theorem of Anderson and Schoen, $\partial_{\Delta} M$ is naturally isomorphic to the sphere at infinity and $\partial_{\Delta}^{\min } M=\partial_{\Delta} M$ if $M$ is a complete and simply connected Riemannian manifold with pinched negative sectional curvature AS, $\mathrm{An}, \mathrm{Ki}$. In the case where $M$ is the universal covering space of a closed rank one manifold $N$ of non-positive curvature, the Poisson boundary $\mathcal{P}(M, \Delta)$ is naturally isomorphic to the sphere at infinity of $M$, using again the isomorphism between $\mathcal{P}(M, \Delta)$ and $\mathcal{P}(X, \mu)$, where $X$ is a fibre of $\pi: M \rightarrow N$ and $\mu$ an associated family of Lyons-Sullivan measures on $X$ [BL1]. However, the relation between the Martin boundary $\partial_{\Delta} M$ and the sphere at infinity is unclear. One hope in this 
direction, nurtured by the isomorphism $\partial_{\Delta}^{\min } M=\partial_{\mu}^{\min } X$, is that the latter might be more accessible than $\partial_{\Delta}^{\min } M$.

1.6. Structure of the article. Besides other preliminaries, Section 2 contains short descriptions of Martin and Poisson boundaries. In Section 3 , we describe the $L S$-discretization procedure and its properties. Much of this is a translation of known results about the Laplacian and Brownian motion. However, Theorem 3.31 and Corollary 3.32 about Martin boundaries and positive harmonic functions are also new for the Laplacian and Brownian motion. In Section 4, we apply the results of Section 3 to covering projections and prove Theorems $\mathrm{A}-\mathrm{D}$. The general Theorem 4.2 and Corollary 4.3 about diffusions on covering spaces might be useful in other contexts. In Section [5, we get analogs of Theorems $\mathrm{A}-\mathrm{D}$ and extensions of Theorem $\mathrm{E}-\mathrm{G}$ for properly discontinuous group actions. In Section 6. we obtain analogs of Theorems $\mathrm{A}-\mathrm{G}$ for random walks, that is, Markov chains on countable sets.

\section{Preliminaries}

2.1. Diffusion operators and processes. Let $M$ be a connected manifold and $L$ be an elliptic diffusion operator on $M$, in coordinates of $M$ written as

$$
L f=\frac{1}{2} \sum_{i j} a^{i j} \frac{\partial^{2} f}{\partial x^{i} \partial x^{j}}+\sum_{k} b^{k} \frac{\partial f}{\partial x^{k}},
$$

where the matrix $\left(a^{i j}\right)$ is symmetric and positive definite. A function $h$ on $M$ is said to be $L$-harmonic if $L h=0$.

The inverse of the coefficient matrix $\left(a^{i j}\right)$ is the fundamental matrix of a well defined Riemannian metric on $M$, the Riemannian metric associated to $L$. Its Laplacian $\Delta$ has twice the principal symbol of $-L$, and hence $L+\frac{1}{2} \Delta$ is of first order. Since $L$ and $\Delta$ vanish on constant functions, $L+\frac{1}{2} \Delta$ is a vector field.

We assume that $L$ is symmetric on $C_{c}^{\infty}(M)$ with respect to a smooth volume element on $M$, that is, a measure on $M$ of the form $\varphi^{2} \mathrm{dv}$, where $\varphi$ is a positive smooth function on $M$ and dv the volume element of the Riemannian metric on $M$ associated to $L$. The symmetry of $L$ then implies that

$$
L f=-\frac{1}{2} \Delta f+\langle\nabla \ln \varphi, \nabla f\rangle .
$$

Conversely, any operator of this from is an elliptic diffusion operator on $M$ which is symmetric on $C_{c}^{\infty}(M)$ with respect to the volume element $\varphi^{2} \mathrm{dv}$.

Because of (2.2), the diffusion process $\left(D_{t}\right)$ associated to $L$ is Brownian motion with drift $\nabla \ln \varphi$. We assume throughout that $\left(D_{t}\right)$ has infinite life time since this is a consequence of the assumptions in our applications, namely the existence of recurrent subsets. We will encounter different models of $\left(D_{t}\right)$, but usually we view it as defined on the space $\Omega$ of continuous paths $\omega: \mathbb{R}_{\geq 0} \rightarrow M$ with $D_{t}(\omega)=\omega(t)$ and associated family of probability measures $\left(P_{x}\right)_{x \in M}$ on $\Omega$. We have $P_{x}[\omega(0)=x]=1$. Moreover, the distribution of $P_{x}$ at time $t>0$ has a smooth density $p_{t, x}=p_{t}(x, y)$ with respect to $\varphi^{2} \mathrm{dv}$ which is symmetric in $x, y$. For a measure $\mu$ on $M$, we set

$$
P_{\mu}=\int_{M} \mu(d x) P_{x} .
$$

Recall that $\left(D_{t}\right)$ is a strong Markov process and that, for any $f \in C_{c}^{\infty}(M)$,

$$
f \circ D_{t}-f \circ D_{0}-\int_{0}^{t}\left((L f) \circ D_{s}\right) d s
$$

is a martingale. 
2.2. Martin boundary. The Martin boundary is defined when the diffusion process is transient, that is, when its Green function $G(x, y)<\infty$ for all $x \neq y$ in $M$. Recall that $G(., y)$ is an $L$-superharmonic function which is $L$-harmonic on $M \backslash\{y\}$. Fix $x_{0} \in M$ and define the Martin kernel functions $K(., y)$ by

$$
K(x, y)=\frac{G(x, y)}{G\left(x_{0}, y\right)}
$$

A sequence $\left(y_{n}\right)$ in $M$ is said to converge to a Martin boundary point $\eta$ of $M$ if $d\left(x_{0}, y_{n}\right) \rightarrow \infty$ and if $K\left(., y_{n}\right)$ converges (pointwise) to a harmonic function on $M$. The limit function is then denoted by $K(., \eta)$ and is identified with $\eta$, and the space of limit functions is denoted by $\partial_{L} M$. The Martin compactification $\mathcal{M}(M, L)=M \cup \partial_{L} M$, where points $y \in M$ are identified with the Martin kernel $K(., y)$. Then $\mathcal{M}(M, L)$ together with pointwise convergence is a compact Hausdorff space with $M$ homeomorphically embedded.

Note that $K(., \eta)$ is positive with $K\left(x_{0}, \eta\right)=1$. Any minimal positive harmonic function is a Martin boundary point. Moreover, for any positive $L$-harmonic function $h$ on $M$ with $h\left(x_{0}\right)=1$, there is a unique probability measure $\pi$ on the minimal part $\partial_{L}^{\min } M$ of the Martin boundary $\partial_{L} M$, the part of $\partial_{L} M$ consisting of minimal positive $L$-harmonic functions, such that

$$
h(x)=\int_{\partial_{L}^{\min } M} K(x, \eta) \pi(d \eta) .
$$

We will also need Martin boundaries $\partial_{\mu} X$ of random walks on countable sets $X$ with family $\mu=\left(\mu_{x}\right)_{x \in X}$ of transition probabilities. The definition and discussion of these is analogous to the above.

2.3. Poisson boundary. Consider a random walk on a countable set $X$ with family $\mu=\left(\mu_{x}\right)_{x \in X}$ of transition probabilities and path space $\Omega=X^{\mathbb{N}_{0}}$. Assume that the random walk is irreducible, that is, for all $x, y$ in $X$, there exist $x_{1}, \ldots, x_{k}$ in $X$ such that

$$
\mu_{x}\left(x_{1}\right) \mu_{x_{1}}\left(x_{2}\right) \ldots \mu_{x_{k-1}}\left(x_{k}\right) \mu_{x_{k}}(y)>0 .
$$

For $k \geq 2$, define a family $\mu^{k}=\left(\mu_{x}^{k}\right)_{x \in X}$ of probability measures on $X$ recursively by

$$
\mu_{x}^{k}(y)=\sum_{z \in X} \mu_{x}(z) \mu_{z}^{k-1}(y)
$$

where $\mu^{1}=\mu$ (and $\mu^{0}=\delta=\left(\delta_{x}\right)_{x \in X}$, the family of Dirac measures on $X$ ). Irreducibility is then equivalent to the property that, for all $x, y \in X$,

$$
\mu_{x}^{k}(y)>0
$$

for some $k \geq 1$.

Endow $X$ with the discrete topology and let $\mathcal{B}_{\Omega}$ be the $\sigma$-algebra of Borel sets of $\Omega$. For $x \in X$, let $P_{x}$ be the probability measure on $\left(\Omega, \mathcal{B}_{\Omega}\right)$ with

$$
\begin{aligned}
P_{x}[\omega(0) & \left.=x_{0}, \omega\left(k_{1}\right) \in A_{1}, \ldots, \omega\left(k_{n}\right) \in A_{n}\right] \\
= & \delta_{x}\left(x_{0}\right) \sum_{x_{1} \in A_{1}} \mu_{x_{0}}^{k_{1}}\left(x_{1}\right) \sum_{x_{2} \in A_{2}} \mu_{x_{1}}^{k_{2}-k_{1}}\left(x_{2}\right) \cdots \sum_{x_{n} \in A_{n}} \mu_{x_{n-1}}^{k_{n}-k_{n-1}}\left(x_{n}\right)
\end{aligned}
$$

for all integers $0<k_{1}<k_{2}<\cdots<k_{n}$ and subsets $A_{1}, \ldots, A_{n}$ of $X$. Let $\mathcal{F}$ be the extension of $\mathcal{B}_{\Omega}$ by subsets $N$ of $\Omega$ which are null sets with respect to all $P_{x}$. Then the measures $P_{x}$ extend naturally to $(\Omega, \mathcal{F})$.

We say that $\omega_{1}, \omega_{2} \in \Omega$ are stationary equivalent if there are $k_{1}, k_{2} \in \mathbb{N}$ such that $\omega_{1}\left(k_{1}+k\right)=\omega_{2}\left(k_{2}+k\right)$ for all $k \in \mathbb{N}$. We say that a measurable subset $A$ of $\Omega$ is stationary mod zero if there are sets $N^{-} \subseteq A$ and $N^{+} \subseteq \Omega \backslash A$, which 
are null sets with respect to all $P_{x}$, such that $\left(A \backslash N^{-}\right) \cup N^{+}$is the union of stationary equivalence classes. The set $\mathcal{S}$ of measurable subsets of $\Omega$ which are stationary mod zero is a $\sigma$-subalgebra of $\mathcal{F}$. By the irreducibility of the random walk, all the measures $P_{x}$ are equivalent on $\mathcal{S}$. We call $\Omega$ together with $\mathcal{S}$ and the restrictions of the measures $P_{x}$ to $\mathcal{S}$ a Poisson boundary of the Markov chain. More generally, if $\pi:(\Omega, \mathcal{S}) \rightarrow(\mathcal{P}, \mathcal{T})$ is a surjection and $\mathcal{P}$ is equipped with the measures $\nu_{x}=\pi_{*} P_{x}$, then we call $\left(\mathcal{P}, \mathcal{T},\left(\nu_{x}\right)_{x \in X}\right)$ a Poisson boundary of the Markov chain if $\pi^{*}: L^{\infty}(\mathcal{P}, \mathcal{T}) \rightarrow L^{\infty}(\Omega, \mathcal{S})$ is an isomorphism. (In other words, given the other conditions, $\pi^{*}: \mathcal{T} \rightarrow \mathcal{S}$ is an isomorphism modulo sets of measure zero.)

A bounded function $f: X \rightarrow \mathbb{R}$ is called $\mu$-harmonic if

$$
f(x)=\sum_{y \in X} \mu_{x}(y) f(y)
$$

for all $x \in X$. For any such function $f$, the family $\left(f \circ X_{k}\right)_{k \in \mathbb{N}_{0}}$ is a bounded martingale, where $X_{k}(\omega)=\omega_{k}$. Therefore, for each $x \in X$,

$$
f_{\infty}(\omega)=\lim _{k \rightarrow \infty} f\left(X_{k}(\omega)\right)
$$

exists for $P_{x}$-almost every $\omega \in \Omega$. If the limit exists for $\omega$, then also for any $\omega^{\prime}$ which is stationary equivalent to $\omega$, and then $f_{\infty}(\omega)=f_{\infty}\left(\omega^{\prime}\right)$. Hence the limit function $f_{\infty}$ is $\mathcal{S}$-measurable. Since all $P_{x}$ are equivalent on $\mathcal{S}, f_{\infty}$ is uniquely defined as an element of $L^{\infty}(\Omega, \mathcal{S})$. Moreover, since $\left(f \circ X_{k}\right)_{k \in \mathbb{N}_{0}}$ is a bounded martingale,

$$
f(x)=\int_{\Omega} f_{\infty}(\omega) P_{x}(d \omega) .
$$

More generally, for any Poisson boundary as above, we get

$$
f(x)=\int_{\mathcal{P}} \varphi(\eta) \nu_{x}(d \eta)
$$

where $\varphi=f_{\infty} \circ \pi$. Conversely, any $f$ defined in this way is a bounded $\mu$-harmonic function on $X$. The above formula is similar to the Poisson formula (2.6), except that here the measures are given, whereas there the kernel functions are given.

2.4. $F C$-hypercenters and $F C$-hypercentral groups. For any group $\Gamma$, let $F C(\Gamma)$ be the union of all elements of $\Gamma$ whose conjugacy classes are finite, the $F C$-center of $\Gamma$, a normal subgroup of $\Gamma$ containing the center of $\Gamma$. The upper $F C$-central series of normal subgroups $\Gamma_{\alpha}$ of $\Gamma$ is defined by

$$
\begin{array}{rlrl}
\Gamma_{0} & =\{e\}, & \\
\Gamma_{\alpha} / \Gamma_{\beta} & =F C\left(\Gamma / \Gamma_{\beta}\right) & & \text { if } \alpha=\beta+1, \\
\Gamma_{\alpha} & =\cup_{\beta<\alpha} \Gamma_{\beta} & & \text { if } \alpha \text { is a limit ordinal, }
\end{array}
$$

where $\alpha$ runs over all ordinals. The series of $\Gamma_{\alpha}$ stabilizes eventually, that is, there is a first ordinal $\alpha$ with $\Gamma_{\alpha}=\Gamma_{\beta}=: \Gamma_{\lim }$ for all $\beta \geq \alpha$, and $\Gamma_{\lim }$ is called the $F C$-hypercenter of $\Gamma$. We say that $\Gamma$ is $F C$ - $\alpha$-hypercentral if $\Gamma=\Gamma_{\alpha}$. Replacing the $F C$ centers by centers, we get the corresponding and more common notions of upper central series, hypercenter, and $\alpha$-hypercentral. Clearly, $\alpha$-hypercentral groups are $F C$ - $\alpha$-hypercentral. If the specific $\alpha$ is irrelevant, we also speak of $F C$ hypercentral or hypercentral groups, respectively. Synonyms in the literature are FC-hypernilpotent and hypernilpotent.

Echterhoff showed that $\Gamma$ is $F C$-hypercentral if and only if it is amenable and every prime ideal of its group $C^{*}$-algebra is maximal $\mathrm{Ec}$, thus providing one reason to non-experts why such groups are interesting. On the other hand, if $\Gamma$ is finitely generated, then $\Gamma$ is $F C$-hypercentral if and only if it is virtually nilpotent. 


\section{LyONS-SULLIVAN DISCRETIZATION}

Following earlier work of Furstenberg, Lyons and Sullivan (LS) constructed a discretization of Brownian motion on Riemannian manifolds LS. The LS-construction actually applies to diffusion processes, and this is one of the objectives of this section. Our presentation of the LS-construction is close to the one in BL2], where the original case of Brownian motion is discussed.

3.1. Balayage and $L$-harmonic functions. Let $F \subseteq M$ be closed and $V \subseteq M$ be open. For $\omega \in \Omega$, the respective hitting and exit time,

$$
\begin{aligned}
& R^{F}(\omega)=\inf \{t \geq 0 \mid \omega(t) \in F\}, \\
& S^{V}(\omega)=\inf \{t \geq 0 \mid \omega(t) \in M \backslash V\},
\end{aligned}
$$

are stopping times. For a Borel subset $A \subseteq M$, let

$$
\begin{aligned}
& \beta(\mu, F)(A)=\beta_{\mu}^{F}(A)=P_{\mu}\left(\omega\left(R^{F}(\omega)\right) \in A\right), \\
& \varepsilon(\mu, V)(A)=\varepsilon_{\mu}^{V}(A)=P_{\mu}\left(\omega\left(S^{V}(\omega)\right) \in A\right),
\end{aligned}
$$

where $\beta$ stands for balayage and $\varepsilon$ for exit. In the case of Dirac measures, $\mu=\delta_{x}$, we use the shorthand $x$ for $\delta_{x}$. If $\beta(x, F)(F)=1$ for all $x \in M$, then $F$ is said to be recurrent. This is equivalent to $R^{F}<\infty$ almost surely with respect to each $P_{x}$.

Proposition 3.3. Let $F$ be a recurrent closed subset of $M, \mu$ a finite measure on $M$, and $h: M \rightarrow \mathbb{R}$ an L-harmonic function. Then we have:

(1) If $h$ is bounded, then $\mu(h)=\beta(\mu, F)(h)$.

(2) If $h$ is positive, then $\beta(\mu, F)(h) \leq \mu(h)$.

Proof. It suffices to consider the case where $\mu=\delta_{x}$. For $x \in F$, we have $\beta(x, F)=$ $\delta_{x}$ and hence that $\beta(x, F)(h)=h(x)$. Let now $x \in M \backslash V$ and

$$
x \in U_{1} \subseteq U_{2} \subseteq U_{3} \subseteq \ldots
$$

be a sequence of relatively compact open subsets exhausting $M \backslash F$. Let $\chi_{n}$ be a cut-off function on $M$ with $U_{n} \subseteq\left\{\chi_{n}=1\right\} \subseteq \operatorname{supp} \chi_{n} \subseteq U_{n+1}$ and set $f_{n}=\chi_{n} h$. Then $f_{n} \in C_{c}^{\infty}(M)$ and hence

$$
Y_{t}=f_{n} \circ X_{t}-f_{n}(x)-\int_{0}^{t}\left(\left(L f_{n}\right) \circ X_{s}\right) d s
$$

is a $P_{x}$-martingale, hence also $Y_{t \wedge \sigma_{n}}$, where $\sigma_{n}$ denotes the exit time from $U_{n}$. Since $f_{n}$ and $h$ coincide on $U_{n}$ and $h$ is $L$-harmonic, we get that $h \circ X_{t \wedge \sigma_{n}}$ is a $P_{x}$-martingale and also that $h \circ X_{t \wedge \sigma_{n}}=E_{x}\left[h \circ X_{\sigma_{n}} \mid \mathcal{F}_{t \wedge \sigma_{n}}\right]$. In particular, $h(x)=$ $E_{x}\left[h \circ X_{\sigma_{n}}\right]$. Now we have the increasing limit $\sigma_{n} \rightarrow \tau$, in particular the convergence $X_{\sigma_{n}(\omega)}(\omega) \rightarrow X_{\tau(\omega)}(\omega)$, where $\tau$ denotes the time of hitting $F$. In the case where $h$ is bounded, the dominated convergence theorem implies that $h(x)=E_{x}\left[h \circ X_{\tau}\right]$. In the case where $h$ is positive, the Fatou lemma gives

$$
\begin{aligned}
h(x)=E_{x}\left[h \circ X_{\sigma_{n}}\right] & =\liminf E_{x}\left[h \circ X_{\sigma_{n}}\right] \\
& \geq E_{x}\left[\liminf \left(h \circ X_{\sigma_{n}}\right)\right]=E_{x}\left[h \circ X_{\tau}\right],
\end{aligned}
$$

where we use that $\left(h \circ X_{\sigma_{n}(\omega)}\right)(\omega) \rightarrow\left(h \circ X_{\tau(\omega)}\right)(\omega)$.

Corollary 3.4. If the diffusion process on $M$ is recurrent, then positive $L$-harmonic functions on $M$ are constant.

Proof. If the diffusion process is recurrent, then any closed ball in $M$ is recurrent. By Proposition 3.3, a minimum of a positive harmonic function $h$ on such a ball is a global minimum of $h$, and hence $h$ is constant, by the maximum principle.

Remark 3.5. The analogue of Corollary 3.4 also holds for random walks. 
An $L$-harmonic function $h$ on $M$ is said to be swept by $F$ if $\beta(x, F)(h)=h(x)$ for all $x \in M$. Then

$$
\mu(h)=\beta(\mu, F)(h) .
$$

for all finite measures $\mu$ on $M$. By Proposition 3.311, any bounded $L$-harmonic function is swept by any recurrent closed subset of $M$.

3.2. $L S$-discretization and $L$-harmonic functions. Let $X$ be a discrete subset of $M$. Families $\left(F_{x}\right)_{x \in X}$ of compact subsets and $\left(V_{x}\right)_{x \in X}$ of relatively compact open subsets of $M$ together with a constant $C>1$ will be called regular Lyons-Sullivan data for $X$ or, for short, $L S$-data for $X$ if

(D1) $x \in \stackrel{\circ}{F}_{x}$ and $F_{x} \subseteq V_{x}$ for all $x \in X$;

(D2) $F_{x} \cap V_{y}=\emptyset$ for all $x \neq y$ in $X$;

(D3) $F=\cup_{x \in X} F_{x}$ is closed and recurrent;

(D4) for all $x \in X$ and $y \in F_{x}$,

$$
\frac{1}{C}<\frac{d \varepsilon\left(y, V_{x}\right)}{d \varepsilon\left(x, V_{x}\right)}<C
$$

We say that $X$ is $*$-recurrent if it admits LS-data. Our requirements (D1) and (D2) are more restrictive than the corresponding ones in [LS] and are conform to the ones in BL2.

Suppose now that we are given LS-data as above. For a finite measure $\mu$ on $M$, define measures

$$
\mu^{\prime}=\sum_{x \in X} \int_{F_{x}} \beta_{\mu}^{F}(d y)\left(\varepsilon_{y}^{V_{x}}-\frac{1}{C} \varepsilon_{x}^{V_{x}}\right) \quad \text { and } \quad \mu^{\prime \prime}=\frac{1}{C} \sum_{x \in X} \int_{F_{x}} \beta_{\mu}^{F}(d y) \delta_{x}
$$

on $M$ with support on $\cup_{x \in X} \partial V_{x}$ and $X$, respectively.

Proposition 3.8. If $h$ is a positive L-harmonic function on $M$ swept by $F$ and $\mu$ is a finite measure on $M$, then

$$
\mu(h)=\mu^{\prime}(h)+\mu^{\prime \prime}(h) \quad \text { and } \quad \mu^{\prime}(h) \leq\left(1-\frac{1}{C^{2}}\right) \mu(h) .
$$

Proof. For the first assertion, we compute

$$
\begin{aligned}
\mu^{\prime}(h) & =\sum_{x \in X} \int_{F_{x}} \beta_{\mu}^{F}(d y)\left(\varepsilon_{y}^{V_{x}}(h)-\frac{1}{C} \varepsilon_{x}^{V_{x}}(h)\right) \\
& =\sum_{x \in X} \int_{F_{x}} \beta_{\mu}^{F}(d y)\left(h(y)-\frac{1}{C} h(x)\right) \\
& =\int_{F} \beta_{\mu}^{F}(d y) h(y)-\mu^{\prime \prime}(h) \\
& =\beta_{\mu}^{F}(h)-\mu^{\prime \prime}(h)=\mu(h)-\mu^{\prime \prime}(h) .
\end{aligned}
$$

Moreover, by (D4), we have

$$
\varepsilon_{y}^{V_{x}}(h)-\frac{1}{C} \varepsilon_{x}^{V_{x}}(h) \leq\left(1-\frac{1}{C^{2}}\right) \varepsilon_{y}^{V_{x}}(h)
$$

for all $x \in X$ and $y \in F_{x}$. This implies the second assertion.

For $y \in M$, let now

$$
\mu_{y, 0}= \begin{cases}\delta_{y} & \text { if } y \notin X \\ \varepsilon\left(y, V_{y}\right) & \text { if } y \in X\end{cases}
$$

and set recursively, for $n \geq 1$,

$$
\mu_{y, n}=\left(\mu_{y, n-1}\right)^{\prime} \quad \text { and } \quad \tau_{y, n}=\left(\mu_{y, n-1}\right)^{\prime \prime} .
$$


The associated $L S$-measure is the probability measure

$$
\mu_{y}=\sum_{n \geq 1} \tau_{y, n}
$$

with support on $X$.

Proposition 3.12. The LS-measures $\mu_{y}$ have the following properties:

(1) $\mu_{y}$ is a probability measure on $X$ such that $\mu_{y}(x)>0$ for all $x \in X$;

(2) for any $x \in X$ and diffeomorphism $\gamma$ of $M$ leaving $L, X$, and the LS-data invariant,

$$
\mu_{\gamma y}(\gamma x)=\mu_{y}(x)
$$

(3) for all $x \in X$,

$$
\mu_{x}=\int_{\partial V_{x}} \varepsilon_{x}^{V_{x}}(d y) \mu_{y}
$$

(4) for all $x \in X$ and $y \in F_{x}$ different from $x$,

$$
\mu_{y}=\frac{1}{C} \delta_{x}+\int_{\partial V_{x}} \varepsilon_{x}^{V_{x}}(d z)\left(\frac{d \varepsilon\left(y, V_{x}\right)}{d \varepsilon\left(x, V_{x}\right)}-\frac{1}{C}\right) \mu_{z} ;
$$

(5) for any $y \in M \backslash F$ and stopping time $T \leq R^{F}$,

$$
\mu_{y}=\int \pi_{y}^{T}(d z) \mu_{z}
$$

where $\pi_{y}^{T}$ denotes the distribution of $P_{y}$ at time $T$.

Proof. For the total mass of the $\mu_{y, n}$, we have $\mu_{y, 0}(M)=1$ and, recursively,

$$
\mu_{y, n}(M) \leq\left(1-\frac{1}{C^{2}}\right) \mu_{y, n-1}(M) \leq\left(1-\frac{1}{C^{2}}\right)^{n} \mu_{y, 0}(M) .
$$

Since

$$
\mu_{y, 0}(M)=\mu_{y, n}(M)+\sum_{1 \leq k \leq n} \tau_{y, k}(M),
$$

we get that $\mu_{y}$ inherits all the mass of $\mu_{y, 0}$ eventually. This shows the first claim in (11). The second is clear since $P_{y}\left[\left\{\omega \in \Omega \mid \omega(t) \in F_{x}\right.\right.$ for some $\left.\left.t>0\right\}\right]>0$ for all $x \in X$. Assertion (2) is obvious. Assertions (3)-(5) follow immediately from the definition of the LS-measures and the strong Markov property of the process, observing that $\beta_{y}^{F}=\delta_{y}$ in (4).

In the case of Brownian motion, the following is Theorem 1.10 of [BL2] (where the reader is referred to the discussion on [LS, p. 317]).

Theorem 3.13. Let $h$ be a positive L-harmonic function on $M$. Then we have:

(1) If $h$ is swept by $F$, then $\mu_{y}(h)=h(y)$ for all $y \in M$.

(2) If $h$ is not swept by $F$, then $\mu_{y}(h)<h(y)$ for all $y \in M$.

Proof. (11) Let $y \in M$. Then $\mu_{y, 0}(h)=h(y)$. With $\tau_{y, 0}=0$, assume by induction that

$$
\mu_{y, n}(h)+\sum_{1 \leq k \leq n} \tau_{y, k}(h)=h(y) \quad \text { with } \quad \mu_{y, n}(h) \leq\left(1-\frac{1}{C^{2}}\right)^{n} h(y)
$$


By Proposition 3.8. applied to $\mu=\mu_{y, n}$, we then get

$$
\begin{aligned}
h(y) & =\mu_{y, n}(h)+\sum_{1 \leq k \leq n} \tau_{y, k}(h) \\
& =\mu_{y, n+1}(h)+\tau_{y, n+1}(h)+\sum_{1 \leq k \leq n} \tau_{y, k}(h) \\
& =\mu_{y, n+1}(h)+\sum_{1 \leq k \leq n+1} \tau_{y, k}(h)
\end{aligned}
$$

with

$$
\mu_{y, n+1}(h) \leq\left(1-\frac{1}{C^{2}}\right) \mu_{y, n}(h) \leq\left(1-\frac{1}{C^{2}}\right)^{n+1} h(y) .
$$

Hence (3.14) holds for all $n$. The asserted equality $\mu_{y}(h)=h(y)$ is an immediate consequence.

(2) If $h$ is not swept by $F$, there is a point $z_{0} \in M \backslash F$ such that $\beta\left(z_{0}, F\right)(h)<$ $h\left(z_{0}\right)$. Then, by the strong Markov property of the process, $\beta(z, F)(h)<h(z)$ for all $z$ in the connected neighborhood $U$ of $M \backslash F$ containing $z_{0}$. Now starting at any $y \in M$, the probability of entering $U$ at some positive time is positive.

Denote by $\mathcal{H}^{+}(M, L), \mathcal{H}^{\infty}(M, L), \mathcal{H}^{+}(X, \mu)$, and $\mathcal{H}^{+}(X, \mu)$ the spaces of positive and bounded $L$-harmonic and $\mu$-harmonic functions on $M$ and $X$, respectively. Let $\mathcal{H}_{F}^{+}(M, L)$ be the space of positive $L$-harmonic functions on $M$ swept by $F$.

Theorem 3.15. The restriction map $\mathcal{H}_{F}^{+}(M, L) \rightarrow \mathcal{H}^{+}(X, \mu)$ is an isomorphism. In particular, the restriction map $\mathcal{H}^{\infty}(M, L) \rightarrow \mathcal{H}^{\infty}(X, \mu)$ is an isomorphism.

In the case of Brownian motion, Theorem 3.15] is Theorem 1.11 of BL2]. Mutatis mutandis, the proof in BL2] carries over to our more general setting.

3.3. $L S$-path discretization and Poisson boundary. Let $\left(F_{x}, V_{x}\right)$ be $L S$-data for $X$ and $\left(\mu_{y}\right)_{y \in M}$ be the associated family of $L S$-measures on $X$. For $\omega \in \Omega$, set

$$
S_{0}(\omega)= \begin{cases}0 & \text { if } \omega(0) \notin X, \\ S_{0}^{V_{x}}(\omega) & \text { if } \omega(0)=x \in X,\end{cases}
$$

and recursively, for $n \geq 1$,

$$
\begin{aligned}
& R_{n}(\omega)=\inf \left\{t \geq S_{n-1}(\omega) \mid \omega(t) \in F\right\}, \\
& S_{n}(\omega)=\inf \left\{t \geq R_{n}(\omega) \mid \omega(t) \notin V_{X_{n}(\omega)}\right\},
\end{aligned}
$$

where $X_{n}=X_{n}(\omega) \in X$ with $Y_{n}=Y_{n}(\omega)=\omega\left(R_{n}(\omega)\right) \in F_{X_{n}(\omega)}$.

On $\tilde{\Omega}=\Omega \times[0,1]^{\mathbb{N}}$, let $N_{0}(\omega, \alpha)=0$ and recursively, for $k \geq 1$,

$$
N_{k}(\omega, \alpha)=\inf \left\{n>N_{k-1}(\omega, \alpha) \mid \alpha_{n}<\kappa\left(X_{n}(\omega), Y_{n}(\omega), Z_{n}(\omega)\right)\right\}
$$

where we write $\alpha=\left(\alpha_{1}, \alpha_{2}, \ldots\right) \in[0,1]^{\mathbb{N}}$ and $Z_{n}=Z_{n}(\omega)=\omega\left(S_{n}(\omega)\right)$ and where

$$
\kappa=\kappa(x, y, z)=\frac{1}{C} \frac{d \varepsilon\left(x, V_{x}\right)}{d \varepsilon\left(y, V_{x}\right)}(z)
$$

for $x \in X, y \in F_{x}$ and $z \in \partial V_{x}$. We also set

$$
T_{k}(\omega, \alpha)=S_{N_{k}(\omega, \alpha)}(\omega) .
$$

For $y \in M$, let $\tilde{P}_{y}$ be the product measure $P_{y} \otimes \lambda^{\mathbb{N}}$ on $\tilde{\Omega}$, where $\lambda$ denotes the Lebesgue measure on $[0,1]$. Arguing as in [LS, Chapter 8], we arrive at the following two results. 
Lemma 3.21. For $y \in M$, the distribution of $\tilde{P}_{y}$ at time $T_{1}$ is given by

$$
\sum_{x \in X} \mu_{y}(x) \varepsilon_{x}^{V_{x}}
$$

In particular, for all $x \in X$, we have $\tilde{P}_{y}\left[X_{N_{1}}=x\right]=\mu_{y}(x)$.

Proof. View $\omega \in \Omega$ as having mass one initially and that it loses

$$
\kappa_{n}=\kappa\left(X_{n}(\omega), Y_{n}(\omega), Z_{n}(\omega)\right)
$$

times its remaining mass to the corresponding points $x \in X$ when entering there domains $F_{x}$ at the hitting times $R_{n}$.

By the strong Markov property of the process, the distribution of $P_{y}$ at time $S_{1}$ is given by

$$
\sum_{x \in X} \int_{F_{x}} \beta_{y}^{F}(d u) \varepsilon_{u}^{V_{x}} \text { and } \sum_{x \in X} \int_{F_{x}} \beta_{\varepsilon_{y}}^{F}(d u) \varepsilon_{u}^{V_{x}}
$$

for $y \in M \backslash X$ and $y \in X$, respectively, where $\varepsilon_{y}$ stands for $\varepsilon\left(y, V_{y}\right)$ in the latter case. Now for each $\omega \in \Omega$, exactly a part $\kappa\left(X_{1}(\omega), Y_{1}(\omega), Z_{1}(\omega)\right)$ of $(\omega, \alpha) \in \tilde{\Omega}$ satisfy $N_{1}(\omega, \alpha)=1$. The distribution of the part $N_{1}=1$ of $\tilde{\Omega}$ at time $S_{1}$ is therefore

$$
\left.\begin{array}{l}
\frac{1}{C} \sum_{x \in X} \int_{F_{x}} \beta_{y}^{F}(d u) \frac{d \varepsilon_{x}^{V_{x}}}{d \varepsilon_{u}^{V_{x}}} \varepsilon_{u}^{V_{x}}=\frac{1}{C} \sum_{x \in X} \beta_{y}^{F}\left(F_{x}\right) \varepsilon_{x}^{V_{x}} \\
\frac{1}{C} \sum_{x \in X} \int_{F_{x}} \beta_{\varepsilon_{y}}^{F}(d u) \frac{d \varepsilon_{x}^{V_{x}}}{d \varepsilon_{u}^{V_{x}}} \varepsilon_{u}^{V_{x}}=\frac{1}{C} \sum_{x \in X} \beta_{\varepsilon_{y}}^{F}\left(F_{x}\right) \varepsilon_{x}^{V_{x}}
\end{array}\right\}=\sum_{x \in X} \tau_{y, 1}(x) \varepsilon_{x}^{V_{x}}
$$

for $y \in M \backslash X$ and $y \in X$, respectively. The distribution of the remaining part $N_{1}>1$ of $\tilde{\Omega}$ at time $S_{1}$ is then given by $\mu_{y, 1}=\left(\mu_{y, 0}\right)^{\prime}$. Using the strong Markov property, we obtain recursively that the distribution of the parts $N_{1} \leq k$ and $N_{1}>k$ of $\tilde{\Omega}$ at time $S_{k}$ are given by

$$
\sum_{x \in X} \sum_{1 \leq j \leq k} \tau_{y, j}(x) \varepsilon_{x}^{V_{x}} \text { and }\left(\mu_{y, n}\right)^{\prime},
$$

respectively.

The discussion in [LS, p. 321] applies in our setup as well (see also [BL2, Theorem 2.3] and [K2, Proposition 4]) and gives the following result.

Theorem 3.22. The process $\left(X_{N_{k}}\right)_{k \geq 0}$ is a Markov process with time homogeneous transition probabilities $\mu_{y}(x)$, where $x \in X$ and $y \in M$. More precisely,

$$
\tilde{P}_{y}\left[X_{N_{1}}=x_{1}, \ldots, X_{N_{k}}=x_{k}\right]=\mu_{y}\left(x_{1}\right) \mu_{x_{1}}\left(x_{2}\right) \ldots \mu_{x_{k-1}}\left(x_{k}\right) .
$$

Proof. We show that the distribution of $\tilde{P}_{y}$ restricted to $\left\{X_{N_{1}}=x_{1}, \ldots, X_{N_{k}}=x_{k}\right\}$ at time $T_{k}$ is given by

$$
\mu_{y}\left(x_{1}\right) \mu_{x_{1}}\left(x_{2}\right) \ldots \mu_{x_{k-1}}\left(x_{k}\right) \varepsilon_{x_{k}}^{V_{x_{k}}} .
$$

For $k=1$, this is Lemma 3.21. Assume recursively that the assertion holds for all $j \leq k-1$ for some $k \geq 2$. Then the distribution of $\tilde{P}_{y}$ of the part $\left\{X_{N_{1}}=\right.$ $\left.x_{1}, \ldots, X_{N_{k-1}}=x_{k-1}\right\}$ of $\tilde{\Omega}$ at time $T_{n-1}$ is given by

$$
\sum \mu_{y}\left(x_{1}\right) \mu_{x_{1}}\left(x_{2}\right) \ldots \mu_{x_{k-2}}\left(x_{k-1}\right) \varepsilon_{x_{k-1}}^{V_{x_{k-1}}} \text {. }
$$

Now $x_{k-1} \in X$, and hence the second alternative in the definition of the LSmeasures applies. That is, $\left(X_{N_{1}}\right)$ with initial distribution $\varepsilon\left(x_{k-1}, V_{x_{k-1}}\right)$ has distribution at time $T_{1}$ as given in Lemma 3.21 (with $y$ substituted by $x_{k-1}$ ). Now using the strong Markov property, we arrive at the asserted equality. 
Theorem 3.23. LS-path discretization induces an isomorphism

$$
\mathcal{P}(X, \mu) \rightarrow \mathcal{P}(M, L)
$$

of Poisson boundaries.

We follow the arguments in the proof of the corresponding [K2, Theorem 1].

Proof of Theorem 3.23. As in [K2, Theorem 1], we decompose $L S$-path discretization into the following four measure preserving maps between Markov processes,

$$
\begin{aligned}
\Omega \ni \omega \underset{1}{\rightarrow} & \left(\omega\left(R_{n}(\omega)\right), \omega\left(S_{n}(\omega)\right)\right) \underset{2}{\leftarrow}\left(\omega\left(R_{n}(\omega)\right), \omega\left(S_{n}(\omega)\right), \alpha_{n}\right) \underset{3}{\rightarrow} \\
& \underset{3}{\rightarrow}\left(\omega\left(R_{N_{k}}(\omega)\right), \omega\left(S_{N_{k}}(\omega)\right), \alpha_{N_{k}}\right) \underset{4}{\rightarrow}\left(X_{N_{k}(\omega)}(\omega)\right) \in M \times X^{\mathbb{N}} .
\end{aligned}
$$

Since the $\alpha_{n}$ are i.i.d. and independent of $\omega$, the second map induces an isomorphism of the corresponding Poisson boundaries. Thus we obtain a measurable map $\mathcal{P}(M, L) \rightarrow \mathcal{P}(X, \mu)$. By Theorem 3.15, the spaces of bounded harmonic functions on $M$ and $X$ are isomorphic to each other under this map. Hence it is an isomorphism of Poisson boundaries.

The difference to the argument in $\overline{\mathrm{K} 2}$ is that we can use the isomorphism $\mathcal{H}^{\infty}(M, L) \rightarrow \mathcal{H}^{\infty}(X, \mu)$ from Theorem [3.15, whereas in [K2], the aim of the proof is to establish the latter.

3.4. Green functions and Martin boundary. For $x \in X$ and $y \in M$, we let

$$
g(y, x)=\delta_{y}(x)+\sum_{k \geq 1} \tilde{P}_{y}\left(X_{N_{k}}=x\right)
$$

the Green function associated to the Markov chain $\left(X_{N_{k}}\right)_{k \geq 1}$.

Proposition 3.25. For all $x \in X$ and $y \in M \backslash V_{x}$, we have

$$
g(y, x)=\frac{1}{C} \sum_{n=1}^{\infty} \nu_{y, n}\left(F_{x}\right)
$$

where $\nu_{y, n}$ denotes the distribution of $P_{y}$ w.r.t. $\omega\left(R_{n}(\omega)\right)$, that is,

$$
\nu_{y, n}(A)=P_{y}\left[\omega\left(R_{n}(\omega)\right) \in A\right] .
$$

In the case of Brownian motion, Proposition 3.25] is Equation 2.5 of [BL2]. Mutatis mutandis, the proof in BL2 carries over to our setting.

The Green function $G$ of the diffusion process $\left(D_{t}\right)$ is given by

$$
G(x, y)=\int_{0}^{\infty} p(t, x, y) d t
$$

The Green function measures the average time of sojourn of a path in subsets of $M$, that is, for any Borel set $B \subset M$, we have

$$
\int_{B} G(x, y) \varphi^{2}(y) \operatorname{dv}(\mathrm{y})=\mathrm{E}_{\mathrm{x}}\left(\int_{0}^{\infty} \chi_{\mathrm{B}}(\omega(\mathrm{t})) \mathrm{dt}\right)
$$

Recall that $G$ is smooth on the set of $(x, y)$ with $x \neq y$.

More generally, for any open subset $V$ of $M$, we let $G_{V}$ be the Green function of $V$. It is obtained by stopping the diffusion process $\left(D_{t}\right)$ upon leaving $V$.

Proposition 3.28. For all $x \in X$ and $y \in M \backslash V_{x}$, we have

$$
G(y, x)=\sum_{n=1}^{\infty} \int_{\partial F_{x}} G_{V_{x}}(z, x) \nu_{y, n}(d z) .
$$


In the case of Brownian motion, Proposition 3.25] is Equation 2.6 of [BL2]. Mutatis mutandis, the proof in BL2] carries over to our setting.

Following [BL2], we say that $L S$-data $\left(F_{x}, V_{x}\right)$ for $X$ are balanced if there is a constant $B$ such that

(D5) $G_{V_{x}}(z, x)=B$ for all $x \in X$ and $z \in \partial F_{x}$. The following result extends [BL2, Theorem 2.7].

Theorem 3.29. If $\left(F_{x}, V_{x}\right)$ are balanced $L S$-data for $X$ with constants $B$ and $C$, then

$$
G(y, x)=B C g(y, x) \quad \text { for all } x \in X \text { and } y \in M \backslash V_{x} .
$$

In particular, the diffusion process $\left(D_{t}\right)$ is transient if and only if the $\mu$-random walk on $X$ is transient. In this case, $\mu_{x}(y)=\mu_{y}(x)$ for all $x, y \in X$.

Proof. The displayed formula follows immediately from Proposition 3.25 and Proposition 3.28. For the assertion about the transience, we recall that $\left(D_{t}\right)$ is transient if and only if $G(x, y)<\infty$ for some-and then all-points $x \neq y$ in $M$ and that the $\mu$-random walk on $X$ is transient if and only if $g(x, y)<\infty$ for some-and then all-points $x, y$ in $X$.

Since $G(x, y)=G(y, x)$ for all $x, y \in X$, we conclude that $g(x, y)=g(y, x)$ for all $x, y \in X$. The proof of the symmetry of $\mu$ is now exactly the same as in the case of Brownian motion in [BL2, Theorem 2.7].

We arrive at the extension of [BL2, Theorem2.8], and the proof there carries over to our setting.

Theorem 3.30. Assume that $M$ is L-transient and that $X$ is endowed with the family $\mu$ of $L S$-measures associated to balanced $L S$-data $\left(F_{x}, V_{x}\right)$. Then the inclusion $X \rightarrow M$ extends to a convex homeomorphism between $\partial_{\mu} X$ and $\partial_{L} M \cap \bar{X}$, where $\bar{X}$ denotes the closure of $X$ in $\mathcal{M}(M, L)$.

We say that $X$ is $*$-uniform if there are families $\left(K_{x} \subseteq U_{x}\right)_{x \in X}$, where the $K_{x}$ are compact and the $U_{x}$ relatively compact and open, such that

(U1) $\cup_{x \in X} K_{x}=M$ and the open covering of $M$ by the $U_{x}$ is locally finite;

(U2) for all $x \in X$ and $y \in K_{x}$,

$$
\frac{1}{C}<\frac{d \varepsilon\left(y, U_{x}\right)}{d \varepsilon\left(x, U_{x}\right)}<C
$$

Here the constant $C>1$, if it exists, is chosen to coincide with the one in (D4) Note that the family of $\left(K_{x}, U_{x}\right)$ can be chosen independently of the $L S$-data.

Theorem 3.31. Assume that $M$ is L-transient, that $X$ is *-uniform, and that $X$ is endowed with the family $\mu$ of $L S$-measures associated to balanced $L S$-data $\left(F_{x}, V_{x}\right)$. Then

(1) the $\mu$-random walk on $X$ is transient and the inclusion $X \rightarrow M$ extends to a homeomorphism

$$
\partial_{\mu}^{\min } X \rightarrow \partial_{L}^{\min } M
$$

(2) $\partial_{L}^{\min } M=\partial_{L} M$ if and only if $\partial_{\mu}^{\min } X=\partial_{\mu} X$.

Proof. Choose the origin $x_{0}$ for the Martin kernels associated to $L$ and $\mu$ in $X$; see (2.5). Then they coincide on all points $(y, x)$ in $M \times X$ with $y \notin V_{x}$ and $x \neq x_{0}$, by Theorem 3.29 .

Choose a family $\left(K_{x}, U_{x}\right)_{x \in X}$ satisfying (U1) and (U2), Let $\eta \in \partial_{L}^{\min } M$ and denote the associated minimal positive $L$-harmonic function on $M$ by $K(., \eta)$. Let $\left(y_{n}\right)$ be a diverging sequence in $M$ converging to $\eta$; that is, we have $K\left(., y_{n}\right) \rightarrow$ 
$K(., \eta)$. Let $\left(x_{n}\right)$ be a sequence in $X$ such that $y_{n} \in K_{x_{n}}$ for all $n$. In a first step we show that $K\left(., x_{n}\right) \rightarrow K(., \eta)$.

Indeed, since the $G(z,$.$) are L$-harmonic on $M \backslash\{z\}$, the uniform Harnack constants imply that, for any $x \in M$,

$$
K\left(x, x_{n}\right)<C^{2} K\left(x, y_{n}\right)
$$

for all sufficiently large $n$. Hence, up to passing to a subsequence, the sequence of $K\left(., x_{n}\right)$ converges to a positive $L$-harmonic function $K(., \xi)$ on $M$ with $K(., \xi) \leq$ $C^{2} K(., \eta)$. Since $K(., \eta)$ is minimal and $K\left(x_{0}, \xi\right)=K\left(x_{0}, \eta\right)=1$, we conclude that $K(., \xi)=K(., \eta)$. Since this holds for any converging subsequence of the sequence of $K\left(., x_{n}\right)$, we get that $K\left(., x_{n}\right) \rightarrow K(., \eta)$ as asserted.

In a next step, we show that $K(., \eta)$ restricts to a minimal positive $\mu$-harmonic function on $X$. By passing to a subsequence of $\left(x_{n}\right)$ if necessary, we conclude first that $\left(K\left(., x_{n}\right)\right)$ converges in the Martin compactification of $X$. Clearly, the limit is $K(., \eta)$ and hence $K(., \eta)$ is $\mu$-harmonic on $X$. From Theorem 3.13, we get that $K(., \eta)$ is $\mu$-harmonic on $M$, that is, that $K(y, \eta)=\mu_{y}(K(., \eta))$ for all $y \in M$. Suppose now that $f$ is a $\mu$-harmonic function on $X$ such that $f \leq K(., \eta)$ on $X$. Then the $L$-harmonic extension $h$ of $f$ to $M$, given by $h(y)=\mu_{y}(f)$, satisfies

$$
h(y)=\mu_{y}(f) \leq \mu_{y}(K(., \eta))=K(y, \eta)
$$

for all $y \in M$. Since $K(., \eta)$ is a minimal positive $L$-harmonic function on $M$, this implies that $h=c K(., \eta)$ on $M$. But then $f=\left.h\right|_{X}=c K(., \eta)$ on $X$. Hence $K(., \eta)$ is a minimal positive $\mu$-harmonic function on $X$. In this sense, $\partial_{L}^{\min } M \subseteq \partial_{\mu}^{\min } X$.

Conversely, the extension of a minimal positive $\mu$-harmonic function on $X$ is a minimal positive $L$-harmonic function on $M$, since otherwise it would be a nontrivial integral against some measure supported on $\partial_{L}^{\min } M$. But then the same integral would apply to the restriction to $X$, by the first part of the proof. This is a contradiction.

As for (2), suppose that $\partial_{\mu}^{\min } X=\partial_{\mu} X$. Consider a diverging sequence of points $y_{n} \in M$ such that $K\left(., y_{n}\right) \rightarrow K(., \eta) \in \partial_{L} M$. Choose $x_{n} \in X$ with $y_{n} \in K_{x_{n}}$. Then the sequence of $x_{n}$ is diverging and, up to passing to a subsequence, we have

$$
K\left(., x_{n}\right) \rightarrow K(., \xi) \in \partial_{\mu} X=\partial_{\mu}^{\min } X .
$$

On $X$, we have $K(., \eta) \leq C^{2} K(., \xi)$, and hence $K(., \eta)=K(., \xi)$ on $X$ since $K(., \xi)$ is minimal and $K\left(x_{0}, \eta\right)=K\left(x_{0}, \xi\right)=1$. But then $K(., \eta)$ is minimal on $M$ by what we proved above, and therefore $\partial_{L}^{\min } M=\partial_{L} M$. The proof of the other direction is similar.

Since any positive $L$-harmonic function on $M$ and positive $\mu$-harmonic function on $X$ can be written as an average of minimal positive $L$-harmonic and $\mu$-harmonic functions, respectively, Theorem 3.31 has the following consequence.

Corollary 3.32. Assume that $M$ is L-transient, that $X$ is *-uniform, and that $X$ is endowed with the family $\mu$ of $L S$-measures associated to balanced LS-data $\left(F_{x}, V_{x}\right)$. Then

(1) for any $h \in \mathcal{H}^{+}(M, L)$, the restriction $\left.h\right|_{X}$ of $h$ to $X$ belongs to $\mathcal{H}^{+}(X, \mu)$. More precisely, $h(y)=\mu_{y}(h)$ for all $y \in M$;

(2) the restriction map

$$
\mathcal{H}^{+}(M, L) \rightarrow \mathcal{H}^{+}(X, \mu),\left.\quad h \mapsto h\right|_{X},
$$

is an isomorphism of cones. 


\section{DifFusion PROCESSES AND COVERING PROJECTIONS}

Let $\pi: M \rightarrow N$ be a covering of connected manifolds with group $\Gamma$ of covering transformations. We do not assume that $\pi$ is normal, that is, that the action of $\Gamma$ is transitive on the fibers of $\pi$. Let $L_{0}$ be an elliptic diffusion operator on $N$ and $L$ be the pull back of $L_{0}$ to $M$. Let $\left(E_{t}\right)_{t \geq 0}$ be the diffusion process on $N$ associated to $L_{0}$. For simplicity we assume here that $\left(E_{t}\right)$ has infinite life time. Then we may view $\left(E_{t}\right)$ as the evaluation at time $t \geq 0$ on the space $\Omega_{N}$ of continuous paths $\omega: \mathbb{R}_{\geq 0} \rightarrow N$ together with a family of probability measures $\left(Q_{y}\right)_{y \in N}$ on $\Omega_{N}$ which are determined by their distributions $Q_{y}[\omega(t) \in A], A \in \mathcal{B}(N)$, at times $t \geq 0$, where $\mathcal{B}(N)$ denotes the $\sigma$-algebra of Borel sets in $N$.

By the path lifting property of $\pi$, the map

$$
H:\left\{(x, \omega) \in M \times \Omega_{N} \mid \pi(x)=\omega(0)\right\} \rightarrow \Omega_{M}, \quad H(x, \omega)=\omega_{x},
$$

where $\omega_{x}$ denotes the continuous lift of $\omega$ to $M$ starting at $x$, is a homeomorphism with respect to the compact-open topologies. In what follows, we identify $\Omega_{M}$ accordingly. Evaluation $D_{t}$ at time $t \geq 0$ on $\Omega_{M}$ is then given by $D_{t}(x, \omega)=\omega_{x}(t)$.

Theorem 4.2. For $x \in M$ with $\pi(x)=y$, define the probability measure $P_{x}$ on $\Omega_{M}$ by

$$
P_{x}[A]=Q_{y}[\{\omega \mid(x, \omega) \in A\}], \quad A \in \mathcal{B}\left(\Omega_{M}\right) .
$$

Then $\left(D_{t}\right)_{t \geq 0}$ together with the probability measures $\left(P_{x}\right)_{x \in M}$ is the diffusion process on $M$ with generator $L$ on $C_{c}^{\infty}(M)$.

Proof. Let $V \subseteq N$ be a relatively compact and connected open domain with smooth boundary such that a neighborhood $V^{\prime}$ of $\bar{V}$ is evenly covered by $\pi$, and let $U^{\prime}$ be a sheet of $\pi$ over $V^{\prime}$. Then $\pi: U^{\prime} \rightarrow V^{\prime}$ is a diffeomorphism with $\pi^{*} L_{0}=L$. Hence the diffusion processes associated to $L$ on $U=\pi^{-1}(V) \cap U^{\prime}$ and $L_{0}$ on $V$, started at any $x \in U$ respectively $y=\pi(x) \in V$, correspond to each other until exiting $U$ respectively $V$. By the strong Markov property of diffusion processes, this implies that $\left(D_{t}\right)$ is the diffusion associated to $L$.

Corollary 4.3. For all $x \in M$ with $\pi(x)=y$, we have

$$
Q_{y}[\omega(t) \in A]=P_{x}\left[\omega(t) \in \pi^{-1}(A)\right], \quad A \in \mathcal{B}(N) .
$$

In particular, if the distributions of $D$ and $E$ are given by densities $p=p(t, x, u)$ on $M$ and $q=q(t, y, v)$ on $N$ with respect to associated smooth volume elements on $M$ and $N$, then

$$
q(t, y, v)=\sum_{u \in \pi^{-1}(v)} p(t, x, u)
$$

From now on, we assume that $N$ is $L_{0}$-recurrent, that is, that the diffusion process on $N$ associated to $L_{0}$ is recurrent. We choose $z_{0} \in N$ and let $X=\pi^{-1}\left(z_{0}\right)$, a discrete subset of $M$.

(C) We let $F_{0} \subseteq V_{0}$ be connected neighborhoods of $z_{0}$ in $N$ such that $F_{0}$ is compact, $V_{0}$ is open and relatively compact, and $V_{0}$ is evenly covered by $\pi$. For $x \in X$, we let $F_{x} \subseteq V_{x}$ be the connected components of $F=\pi^{-1}\left(F_{0}\right)$ and $V=\pi^{-1}\left(V_{0}\right)$ containing $x$.

Any such family $\left(F_{x}, V_{x}\right)_{x \in X}$, is $\Gamma$-equivariant, that is, $\gamma F_{x}=F_{\gamma x}$ and $\gamma V_{x}=V_{\gamma x}$ for all $\gamma$ in the group $\Gamma$ of covering transformations of $\pi$.

Lemma 4.4. If $N$ is $L_{0}$-recurrent, then

(1) any family $\left(F_{x}, V_{x}\right)$ satisfying $(C)$ constitutes LS-data for $X$, and the associated LS-measures are $\Gamma$-equivariant.

(2) $X$ admits balanced $L S$-data satisfying $(C)$. 
Moreover, if $N$ is compact, then $X$ is $*$-uniform.

Proof. 1) Since $N$ is connected and $L_{0}$-recurrent, the diffusion process on $N$ associated to $L_{0}$ started at any point of $N$ will hit $F_{0}$ with probability one. Hence its lift started at any point of $M$ will hit the preimage $F$ of $F_{0}$ with probability one. Therefore $F$ is recurrent. The existence of a uniform Harnack constant is clear.

22) Let $V_{0}$ be a connected and relatively compact open neighborhood of $z_{0}$ in $N$ which is evenly covered by $\pi$. Let $G_{V_{0}}$ be the Green function of $V_{0}$ for the operator $L_{0}$. Then $G_{V_{0}}\left(z_{0},.\right)$ vanishes on the boundary of $V_{0}$ and $G_{V_{0}}\left(z_{0}, z\right)$ tends to infinity as $z \in V_{0}$ tends to $z_{0}$. Moreover, under $\pi, G_{V_{0}}$ corresponds to the Green functions $G_{V_{x}}$ of the $V_{x}$. Since $V_{0}$ is evenly covered, we have $V_{x} \cap V_{y}=\emptyset$ for all $x \neq y$ in $X$. Hence for any constant $B>0$, the connected components $F_{x}$ of $\left\{G_{V_{x}} \geq B\right\}$ containing $x$ together with the $V_{x}$ are balanced $L S$-data that satisfy (C).

It remains to prove the last assertion. Now the Riemannian metric on $N$ associated to $L_{0}$ is complete since $M$ is compact. Its pull back to $M$ is the Riemannian metric on $M$ associated to $L$. For $x \in X$, let $K_{x}$ be the closed ball of radius $\operatorname{diam} M$ around $x$ and $U_{x}$ the open ball of radius diam $M+1$. Since the curvature of $M$ is uniformly bounded, the pairs $\left(K_{x}, U_{x}\right)$ have a uniform Harnack constant as required; see [CY, Theorem 6].

Proof of Theorems $A-D$ in the introduction. Theorem $A$ and Theorem $B$ are immediate consequences of Theorem 3.31 and Corollary [3.32, using that $X$ is *uniform and choosing balanced $L S$-data satisfying (C) (using Lemma 4.422).

For the first three assertions of Theorem [C, we need $L S$-data satisfying $(\mathrm{C})$, not necessarily balanced. With such data, Assertion 1 is a consequence of Theorem 3.15, using that bounded harmonic functions are swept by $F$ (Proposition 3.3). Assertion 2 follows from Theorem 3.13 and Assertion 3 from the second assertion in Theorem 3.15, Assertion 4 follows from Theorem 3.29, where we now choose balanced $L S$-data satisfying (C) (using Lemma 4.412).

Assertion 1 of Theorem $D$ follows from Theorem 3.23 with any choice of $L S$ data satisfying (C) Assertion 2 is a consequence of Theorem 3.30, where we choose balanced $L S$-data satisfying (C) (using Lemma 4.4/2).

\section{DifFusion PROCESSES AND PROPERLY DISCONTINUOUS ACTIONS}

In the case of a covering $\pi: M \rightarrow N$ considered in Section 4, the group $\Gamma$ of covering transformations acts properly discontinuously and freely on $M$ and the diffusion operator $L$ on $M$ is the pull back of a diffusion operator on $N$. The action of $\Gamma$ on the fibers of $\pi$ is transitive if the covering is normal. We consider now the case of a properly discontinuous action of a group $\Gamma$ on $M$, extending the case of normal coverings. As before, we let $L$ be a diffusion operator on $M$ which is symmetric with respect to a volume element $\varphi^{2} \mathrm{dv}$, where $\varphi$ is a positive smooth function on $M$ and $\mathrm{dv}$ the volume element of the Riemannian metric on $M$ associated to $L$. We assume that $L$ and $\varphi$ are $\Gamma$-invariant. Without loss of generality, we also assume that the action of $\Gamma$ on $M$ is effective.

We say that $M$ is $L$-recurrent $\bmod \Gamma$ if the stochastic process on $\Gamma \backslash M$ induced by the $L$-diffusion on $M$ is recurrent. Clearly, $L$-recurrence $\bmod \Gamma$ is equivalent to the property that any $\Gamma$-invariant neighborhood of any orbit $\Gamma x, x \in M$, is recurrent with respect to the $L$-diffusion.

We assume from now on now that $M$ is $L$-recurrent $\bmod \Gamma$. We let $x_{0} \in M$ be a point with trivial isotropy group and $X=\Gamma x_{0}$ be its $\Gamma$-orbit.

(A) We let $F_{0} \subseteq V_{0}$ be connected neighborhoods of $x_{0}$ such that $F_{0}$ is compact, $V_{0}$ is open and relatively compact, and $\gamma F_{0} \cap V_{0}=\emptyset$ for all $\gamma \in \Gamma$ not equal to $e$. For $x=\gamma x_{0}$, we let $F_{x}=\gamma F_{0}$ and $V_{x}=\gamma V_{0}$. 
Except for Assertion 3, which is an immediate consequence of Proposition 3.12 the proof of the following lemma is very similar to the proof of Lemma 4.4 and will therefore be omitted.

Lemma 5.1. If $M$ is $L$-recurrent $\bmod \Gamma$, then

(1) any family $\left(F_{x}, V_{x}\right)$ satisfying $(A)$ constitutes $L S$-data for $X$, and the associated $L S$-measures are $\Gamma$-equivariant.

(2) $X$ admits balanced $L S$-data satisfying $(A)$.

(3) under the identification $X \cong \Gamma x_{0}$ via the orbit map, the measures $\mu_{x}$ correspond to the left translates of a probability measure $\nu$ on $\Gamma$ with $\operatorname{supp} \nu=\Gamma$.

Moreover, if the action of $\Gamma$ on $M$ is uniform, then $X$ is *-uniform.

The proofs of the next assertions are very similar to the ones of Theorems AD] at the end of Section 4 and will therefore be omitted.

Theorem A' - D'. The assertions of Theorems $A$ and $B$ hold if $\Gamma \backslash M$ is compact, the assertions of Theorems $\square$ and $D$ if $M$ is L-recurrent mod $\Gamma$.

We now come to the extensions of Theorems $\mathrm{E}, \mathrm{F}$, and $\mathrm{G}$.

Theorem E'. If $M$ is L-recurrent $\bmod \Gamma$ and $h$ is a minimal positive L-harmonic function on $M$, then $h$ is constant or $\gamma^{*} h / h$ is unbounded for some $\gamma \in \Gamma$.

Proof. If there is a constant $b(\gamma)$ such that $\gamma^{*} h / h \leq b(\gamma)$, then $\gamma^{*} h / h=c(\gamma)$ for some constant $c(\gamma)$. Hence, if there is no $\gamma \in \Gamma$ such that $\gamma^{*} h / h$ is unbounded, there is a map $c: \Gamma \rightarrow \mathbb{R}^{+}$such that $\gamma^{*} h / h=c(\gamma)$ for all $\gamma \in \Gamma$. Clearly, $c$ is a homomorphism.

We let $x_{0} \in M$ be a point with trivial isotropy group and normalize $h$ so that $h\left(x_{0}\right)=1$. Then $h\left(\gamma x_{0}\right)=c(\gamma)$ for all $\gamma \in \Gamma$.

We may assume that $M$ is $L$-transient and choose balanced $L S$-data satisfying $(\mathrm{A})$. Then the $L S$-measures on $X=\Gamma x_{0}$ are symmetric. Setting $\nu(\gamma)=\mu_{x_{0}}\left(\gamma x_{0}\right)$, we obtain a symmetric probability measure $\nu$ on $\Gamma$.

Since $\operatorname{ker} c$ contains all elements of $\Gamma$ of order two, we can write $\Gamma \backslash \operatorname{ker} c=B \cup B^{-1}$ as a disjoint union. Using that $\left.h\right|_{X}$ is $\mu$-superharmonic, by Theorem 3.13, and that $c$ is a homomorphism, we get that

$$
\begin{aligned}
0 & =c(1)-1 \\
& \geq \nu(c)-1 \\
& =\sum_{\gamma \in \Gamma} \nu(\gamma)(c(\gamma)-1) \\
& =\sum_{\gamma \in B}\left(\nu(\gamma) c(\gamma)+\nu\left(\gamma^{-1}\right) c\left(\gamma^{-1}\right)-2\right) \\
& =\sum_{\gamma \in B} \nu(\gamma)\left(c(\gamma)+c(\gamma)^{-1}-2\right) \geq 0
\end{aligned}
$$

and hence that $c=1$. Therefore $h$ is constant on $X=\Gamma x_{0}$. It now follows from Theorem 3.13 that $h$ attains a minimum along $X$. But then $h$ is constant, by the maximum principle.

Theorem F'. If $M$ is $L$-recurrent mod $\Gamma$, then any bounded L-harmonic function on $M$ is invariant under the $F C$-hypercenter of $\Gamma$. In particular, if $\Gamma$ is $F C$ hypercentral, then any bounded L-harmonic function on $M$ is constant.

Proof of Theorem $F$. Choose $X=\Gamma x_{0}$ as above and $L S$-data satisfying (A), Then $X$ and the associated family $\mu$ of $L S$-measures are $\Gamma$-invariant. Let $h$ be a bounded $L$-harmonic function on $M$. Then $\left.h\right|_{X}$ is $\mu$-harmonic and therefore invariant under 
the $F C$-hypercenter of $\Gamma$, by Theorem F" But then $h$ is also invariant under the $F C$-hypercenter of $\Gamma$, by Theorem $\mathrm{C}$. If $\Gamma$ is $F C$-hypercentral, then $h$ is constant, again by Theorem $\mathrm{F}^{\prime \prime}$ and Theorem $\mathrm{C} 3$.

Theorem G'. If $M$ is L-recurrent $\bmod \Gamma$, then there is a $\Gamma$-invariant bounded projection $L^{\infty}(M) \rightarrow \mathcal{H}^{\infty}(M, L)$. In particular, if all bounded L-harmonic functions on $M$ are constant, then $\Gamma$ is amenable.

Proof. For $f \in L^{\infty}(M)$, let $f^{\prime}=A\left(\left.f\right|_{X}\right)$, where $A: L^{\infty}(X) \rightarrow \mathcal{H}^{\infty}(X, \mu)$ is the projection from Theorem G', Then $f^{\prime}$ is the restriction of a unique bounded $L$ harmonic function $H f$ on $M$, and $H$ is the required projection.

\section{RANDOM WALKS AND HARMONIC FUNCTIONS}

In what follows, we consider random walks, that is, Markov chains on countable sets. Let $\nu$ be a random walk on $Y$, given by a family $\left(\nu_{y}\right)_{y \in Y}$ of probability measures on $Y$. We let $\left(P_{y}\right)_{y \in Y}$ be the associated family of probability measures on the sample space $\Omega=Y^{\mathbb{N}_{0}}$. We say that $\nu$ is symmetric if

$$
\nu_{y}(z)=\nu_{z}(y) \text { for all } y, z \in Y \text {. }
$$

We say that $\nu$ is irreducible if, for all $y, z \in Y$, there exist $y_{1}, \ldots, y_{k} \in Y$ such that

$$
\nu_{y}\left(y_{1}\right) \nu_{y_{1}}\left(y_{2}\right) \ldots \nu_{y_{k-1}}\left(y_{k}\right) \nu_{y_{k}}(z)>0
$$

For $k \geq 2$, we define a family $\nu^{k}=\left(\nu_{y}^{k}\right)_{y \in Y}$ of probability measures on $Y$ by

$$
\nu_{y}^{k}(z)=\sum_{x \in Y} \nu_{y}(x) \nu_{x}^{k-1}(z)
$$

where $\nu^{1}=\nu$. Irreducibility is then equivalent to the property that, for all $y, z \in Y$,

$$
\nu_{y}^{k}(z)>0 \quad \text { for some } k \geq 1 \text {. }
$$

If $\nu$ is symmetric, then also $\nu^{k}$, for any $k \geq 2$.

We assume that a countable group $\Gamma$ acts on $Y$ and that $\nu$ is $\Gamma$-invariant, that is, that we have

$$
\nu_{\gamma y}(\gamma z)=\nu_{y}(z)
$$

for all $y, z \in Y$ and $\gamma \in \Gamma$. Then the $\nu^{k}$ are also $\Gamma$-invariant.

For $X \subseteq Y$ and $\omega \in Y^{\mathbb{N}_{0}}$, we let $R^{X}(\omega)=\inf \left\{k \geq 1 \mid \omega_{k} \in X\right\}$. We say that $X$ is $\nu$-recurrent if $P_{y}\left[R^{X}(\omega)<\infty\right]=1$ for any $y \in Y$. Then the family $\mu=\left(\mu_{y}\right)_{y \in Y}$ of hitting probabilities,

$$
\mu_{y}(x)=P_{y}\left[\omega\left(R^{X}(\omega)\right)=x\right]=\sum_{i \geq 0} \sum_{y_{1}, \ldots, y_{i} \in Y \backslash X} \nu_{y}\left(y_{1}\right) \nu_{y_{1}}\left(y_{2}\right) \ldots \nu_{y_{i}}(x)
$$

where $y \in Y$ and $x \in X$, are probability measures on $X$ and define a random walk with sample space $\Omega=Y \times X^{\mathbb{N}}$. By the definition of $\mu$, we have

$$
\mu_{y}(x)=\nu_{y}(x)+\sum_{z \in Y \backslash X} \nu_{y}(z) \mu_{z}(x)
$$

for all $y \in Y$ and $x \in X$. By the $\nu$-recurrence of $X$, the space of sequences in $Y^{N_{0}}$ which have infinitely many of its members in $X$ has full $P_{y}$-measure for all $y \in Y$, and the subsequences consisting of the corresponding starting members and members belonging to $X$ will be called the $X$-subsequences.

Proposition 6.8. Let $X \subseteq Y$ be a $\Gamma$-invariant $\nu$-recurrent subset. Then the family $\mu$ of hitting probabilities has the following properties:

(1) $\mu$ is $\Gamma$-invariant.

(2) $\mu$ is symmetric on $X$ if $\nu$ is symmetric. 
(3) The Green functions $g$ of $\mu$ and $G$ of $\nu$ satisfy

$$
g(y, x)=G(y, x) \quad \text { for all } y \in Y \text { and } x \in X .
$$

In particular, the $\mu$-random walk on $X$ is transient if and only if the $\nu$ random walk on $Y$ is transient.

Proof. (1) is clear, and (2) follows immediately from (6.6). As for (3), we have

$$
\begin{aligned}
G(y, x) & =\delta_{y}(x)+\sum_{i \geq 1} P_{y}\left[\omega_{i}=x\right] \\
& =\delta_{y}(x)+\sum_{i \geq 0} \sum_{y_{1}, \ldots, y_{i} \in Y} \nu_{y}\left(y_{1}\right) \nu_{y_{1}}\left(y_{2}\right) \ldots \nu_{y_{i}}(x) \\
& =\delta_{y}(x)+\sum_{j \geq 0} \sum_{x_{1}, \ldots, x_{j} \in X} \mu_{y}\left(x_{1}\right) \mu_{x_{1}}\left(x_{2}\right) \ldots \mu_{x_{j}}(x) \\
& =g(y, x),
\end{aligned}
$$

where we use (6.6) to pass from the second to the third line.

We say that the action of $\Gamma$ on $Y$ is cofinite if $\Gamma \backslash Y$ is finite. We say that $Y$ is $\nu$-recurrent $\bmod \Gamma$ if the orbits of $\Gamma$ in $Y$ are $\nu$-recurrent.

Recall that a function $h$ on $X$ is $\mu$-harmonic if it satisfies (1.1) for all $y \in X$. Similarly, a function $h$ on $Y$ is $\nu$-harmonic if it satisfies (1.1) for all $y \in Y$, where $Y$ is substituted for $X$ and $\nu$ for $\mu$. If $h$ is $\mu$-harmonic, then $h$ is $\mu^{k}$-harmonic for all $k \geq 1$, and similarly for $\nu$-harmonic functions.

Theorem A" - D". Substituting $Y$ for $M$ and $\nu$ for $L$ and letting $X$ be an orbit of $\Gamma$ in $Y$, the assertions of Theorems $A$ and $B$ hold if $\Gamma \backslash Y$ is finite, the assertions of Theorems $C$ and $D$ if $Y$ is $\mu$-recurrent mod $\Gamma$. Here LS-path discretization in Theorem [C1 has to be replaced by passage to $X$-subsequences.

Proof. Given a $\mu$-harmonic function $h$ on $X$, we want to show that it is the restriction of a $\nu$-harmonic function on $Y$. To that end, we extend $h$ to $Y$ by setting

$$
h(y)=\sum_{x \in X} \mu_{y}(x) h(x) .
$$

Since $h$ is $\mu$-harmonic on $X$, the extension agrees with the original $h$ on $X$. However, we have to verify that $h$ is well defined on $Y \backslash X$, that is, that the sum on the right hand side is finite for all $y \in Y \backslash X$. Since $\mu_{y}$ is a probability measure on $X$ for all $y \in Y$, this is clear if $h$ is bounded.

Lemma 6.10. For any $y \in Y$, there exist $y_{0} \in X$ and a constant $c>0$ such that

$$
\mu_{y}(x) \leq c \mu_{y_{0}}(x) \quad \text { for all } x \in X .
$$

Proof. We can assume $y \in Y \backslash X$. By the irreducibility of $\nu$, there exist $i \geq 0$, $y_{0} \in X$, and $y_{1}, \ldots, y_{i} \in Y \backslash X$ such that $\nu_{y_{0}}\left(y_{1}\right) \cdots \nu_{y_{i}}(y)>0$. Using (6.7), we get

$$
\nu_{y_{i}}(y) \mu_{y}(x) \leq \sum_{z \in Y \backslash X} \nu_{y_{i}}(z) \mu_{z}(x)=\mu_{y_{i}}(x)-\nu_{y_{i}}(x) \leq \mu_{y_{i}}(x)
$$

for all $x \in X$. Hence $\mu_{y}(x) \leq \mu_{y_{i}}(x) / \nu_{y_{i}}(y)$. Recursively, we get that

$$
\mu_{y}(x) \leq \frac{1}{\nu_{y_{i}}(y) \cdots \nu_{y_{0}}\left(y_{1}\right)} \mu_{y_{0}}(x)
$$

for all $x \in X$. 
We continue with the proof of the theorem. Therefore

$$
\begin{aligned}
\sum_{z \in Y} \nu_{y}(z) h(z) & =\sum_{x \in X . z \in Y} \nu_{y}(z) \mu_{z}(x) h(x) \\
& =\sum_{x, z \in X} \nu_{y}(z) \mu_{z}(x) h(x)+\sum_{x \in X}\left(\mu_{y}(x)-\nu_{y}(x)\right) h(x) \\
& =\sum_{x \in X} \mu_{y}(x) h(x)=h(y),
\end{aligned}
$$

and hence the extension (6.9) of $h$ is $\nu$-harmonic on $Y$.

Suppose first that $\Gamma \backslash Y$ is finite, and fix a finite set $A$ of representatives of the $\Gamma$ orbits in $Y$. Since $\nu$ is irreducible and $A$ is finite, there are a $k \geq 1$ and an $\varepsilon>0$ such that $\nu_{x}^{k}(y) \geq \varepsilon$ for all $x, y \in A$. Since $\nu$ is $\Gamma$-invariant and $\nu$-harmonic functions are also $\nu^{k}$-harmonic, we get that $h(\gamma x) \geq \varepsilon h(\gamma y)$ for all positive $\nu$-harmonic functions $h, \gamma \in \Gamma$, and $x, y \in A$.

Choose an origin $x_{0} \in X \cap A$. By Proposition 6.83, the associated Martin kernels for the $\mu$ and $\nu$-random walks coincide on $Y \times X$,

$$
K(y, x)=\frac{G(y, x)}{G\left(x_{0}, x\right)}=\frac{g(y, x)}{g\left(x_{0}, x\right)}=k(y, x)
$$

for all $y \in Y$ and $x \in X$. Hence if $\left(x_{n}\right)$ is a diverging sequence in $X$ such that $K\left(., x_{n}\right)$ converges to a positive $\nu$-harmonic function $h$ on $Y$, then the restriction of $h$ to $X$ is a positive $\mu$-harmonic function on $X$.

Let now $\left(y_{n}\right)$ be a diverging sequence in $Y$ such that $y_{n} \rightarrow \eta \in \partial_{\nu}^{\min } Y$, that is, $K\left(., y_{n}\right) \rightarrow K(., \eta)$, a minimal positive $\nu$-harmonic function on $Y$. Write $y_{n}=\gamma_{n} a_{n}$ with $\gamma_{n} \in \Gamma$ and $a_{n} \in A$ and set $x_{n}=\gamma_{n} x_{0}$. Then $\left(x_{n}\right)$ is a diverging sequence in $X$. Since $K\left(., x_{n}\right)$ and $K\left(, . y_{n}\right)$ are $\nu$-harmonic away from $x_{n}$ and $y_{n}$, respectively, we get from the above that $K\left(., x_{n}\right) \leq \varepsilon^{-2} K\left(., y_{n}\right)$ away from $x_{n}$ and $y_{n}$, and hence any limit $\xi$ of (a subsequence of) $\left(K\left(., x_{n}\right)\right)$ is a positive $\nu$-harmonic function on $Y$ dominated by $\eta$. Since $\eta$ is minimal and $\eta\left(x_{0}\right)=\xi\left(x_{0}\right)=1$, we conclude that $\xi=\eta$. Since $k=K$ on $Y \times X$, we also conclude that the restriction of $\xi$ to $X$ is $\mu$-harmonic. Therefore the minimal part of $\partial_{\nu} Y$ is contained in $\partial_{\mu} X$. Conversely, by the above, any bounded or positive $\mu$-harmonic function on $X$ extends to a $\nu$-harmonic function on $Y$. It follows now easily that $\partial_{\mu}^{\min } X=\partial_{\nu}^{\min } Y$.

The proofs of the remaining assertions are similar to the previous ones.

Lemma 6.12. If $\Gamma \backslash X$ is finite and $h$ is a positive $\mu$-harmonic function on $X$, then $\gamma^{*} h / h$ is bounded for any $\gamma$ in the center of $\Gamma$.

Proof. Let $\gamma$ belong to the center of $\Gamma$. For all $a \in \Gamma, x \in X$, and $k \geq 1$, we then have

$$
\mu_{a x}^{k}(\gamma a x)=\mu_{a x}^{k}(a \gamma x)=\mu_{x}^{k}(\gamma x) .
$$

Hence if $c>0$ and $k \geq 1$ are such that $\mu_{x}^{k}(\gamma x) \geq c$ for all $x$ in a finite set of representatives of the orbits of $\Gamma$ in $X$, then $\mu_{x}^{k}(\gamma x) \geq c>0$ for all $x \in X$. With such $c$ and $k$, we obtain

$$
h(x)=\sum_{y \in X} \mu_{x}^{k}(y) h(y) \geq \mu_{x}^{k}(\gamma x) h(\gamma x) \geq \operatorname{ch}(\gamma x)
$$

for any $x \in X$, and then $\gamma^{*} h / h \leq 1 / c$.

Together with the $\Gamma$-invariance, symmetry of $\nu$ gives

$$
\nu_{x}\left(\gamma^{-1} x\right)=\nu_{\gamma x}(x)=\nu_{x}(\gamma x),
$$

for all $x \in Y$ (and similarly for $\mu$ on $X$ ). 
Theorem E". Suppose that $\nu$ is symmetric and that $Y$ is $\nu$-recurrent $\bmod \Gamma$. Let $h$ be a minimal positive $\nu$-harmonic function on $Y$. Then either $h$ is constant or there is a $\gamma \in \Gamma$ such that $\gamma^{*} h / h$ is unbounded.

Proof. Let $x_{0} \in Y$ and set $X=\Gamma x_{0}$. Normalize $h$ so that $h\left(x_{0}\right)=1$ and assume that $\gamma^{*} h / h$ is bounded for all $\gamma \in \Gamma$. Then $c(\gamma)=\gamma^{*} h / h$ is a constant for all $\gamma \in \Gamma$. Clearly, $c: \Gamma \rightarrow \mathbb{R}_{>0}$ is a homomorphism. Using that $\left.h\right|_{X}$ is $\mu$-superharmonic (by an analog of Theorem 3.13 and (6.13), we get

$$
\begin{aligned}
0 & =h\left(x_{0}\right)-1 \\
& \geq \mu_{x_{0}}(h)-1 \\
& =\sum_{x \in X} \mu_{x_{0}}(x)(h(x)-1) \\
& =\frac{1}{2\left|\Gamma_{0}\right|} \sum_{\gamma \in \Gamma}\left(\mu_{x_{0}}\left(\gamma x_{0}\right) h\left(\gamma x_{0}\right)+\mu_{x_{0}}\left(\gamma^{-1} x_{0}\right) h\left(\gamma^{-1} x_{0}\right)-2\right) \\
& =\frac{1}{2\left|\Gamma_{0}\right|} \sum_{\gamma \in \Gamma} \mu_{x_{0}}\left(\gamma x_{0}\right)\left(c(\gamma)+c(\gamma)^{-1}-2\right) \geq 0,
\end{aligned}
$$

where $\Gamma_{0}$ denotes the isotropy group of $x_{0}$. It follows that $c(\gamma)=1$ for all $\gamma \in \Gamma$ with $\mu_{x_{0}}\left(\gamma x_{0}\right)>0$. By passing to $\mu^{k}$ for an appropriate $k$ if necessary, the latter can be achieved for each $\gamma \in \Gamma$. Hence $h$ is invariant under $\Gamma$. But then $h$ is constant since $Y$ is $\mu$-recurrent $\bmod \Gamma$.

If $\Gamma$ is Abelian and the action of $\Gamma$ on $Y$ is cofinite, then $\gamma^{*} h / h$ is bounded for all $\gamma \in \Gamma$, by Lemma 6.12. Hence Theorem $\mathrm{E}^{\prime \prime}$ has the following consequence.

Corollary 6.14. If $\Gamma$ is Abelian, $\nu$ is symmetric, and the action of $\Gamma$ on $Y$ is cofinite then any positive $\nu$-harmonic function on $Y$ is constant.

For left-invariant Markov chains on groups, Corollary 6.14 is well known and attributed to Choquet and Deny, who characterize, more generally, solutions $\mu$ of the equation $\nu=\mu * \nu$ for general measures $\nu$ on locally compact Abelian groups CD, Theorem 3].

Example 6.15. The function $e^{x}$ is a positive $\nu$-harmonic function on $\mathbb{Z}$ with respect to the non-symmetric probability measure $\nu$ supported on $\{-1,1\}$ with $\nu(-1)=$ $e /(e+1)$ and $\nu(1)=1 /(e+1)$.

Using Margulis's reduction to the Abelian case [Ma], we have the following consequence of Corollary 6.14.

Corollary 6.16. If $\Gamma$ is nilpotent and $\mu$ an irreducible symmetric probability measure on $\Gamma$, then any positive $\mu$-harmonic function on $\Gamma$ is constant.

We now come to bounded harmonic functions. We will need the following special case of [Li, Theorem 3.9].

Lemma 6.17. Assume that $\Gamma \backslash Y$ is finite. Let $\sigma \in \Gamma$ and $h \in \mathcal{H}^{\infty}(Y, \mu)$. Assume that $h(\gamma \sigma y)=h(\sigma \gamma y)$ for all $\gamma \in \Gamma$ and $y \in Y$. Then $h(\sigma y)=h(y)$ for all $y \in Y$.

Theorem F". If $\Gamma \backslash Y$ is finite, then all bounded $\nu$-harmonic functions on $Y$ are invariant under the $F C$-hypercenter of $\Gamma$. In particular, if $\Gamma$ is $F C$-hypercentral, then $Y$ does not have non-constant bounded $\nu$-harmonic functions.

The arguments in the proof are taken from the proofs of Lemma 2.4 and Corollary 2.5 in [LZ. 
Proof of Theorem F". Let $N$ be a normal subgroup of $\Gamma$ such that all $h \in \mathcal{H}^{\infty}(Y, \nu)$ are $N$-invariant and $\gamma \in \Gamma$ have the property that its conjugacy class in $\Gamma / N$ is finite. Then the centralizer of $\gamma$ in $\Gamma / N$ has finite index in $\Gamma / N$ and, therefore, its preimage $\Gamma^{\prime}$ in $\Gamma$ finite index in $\Gamma$. We have $\gamma \in \Gamma^{\prime}$ and $N \subseteq \Gamma^{\prime}$. Now $\Gamma^{\prime} \backslash Y$ is finite since $\Gamma^{\prime}$ has finite index in $\Gamma$. By definition, $\left[\gamma, \Gamma^{\prime}\right] \subseteq N$, hence any commutator $\gamma \sigma \gamma^{-1} \sigma^{-1}$ with $\sigma \in \Gamma^{\prime}$ leaves any $h \in \mathcal{H}^{\infty}(Y, \nu)$ invariant. But then $\gamma$ leaves all $h \in \mathcal{H}^{\infty}(Y, \nu)$ invariant, by Lemma 6.17 .

Let $N$ now be the normal group of $\gamma \in \Gamma$ which leave all $h \in \mathcal{H}^{\infty}(Y, \nu)$ invariant. Then, by the above, $F C(\Gamma / N)$ is trivial. Hence $\Gamma_{\alpha} \subseteq N$ for any ordinal $\alpha$.

If $\Gamma$ is hypercentral, then $h$ is $\Gamma$-invariant and has therefore at most $|\Gamma \backslash Y|$ many values. In particular, $h$ has a maximum. Hence $h$ is constant, by the maximum principle.

Theorem G". There is a $\Gamma$-invariant bounded projection $L^{\infty}(X) \rightarrow \mathcal{H}^{\infty}(X, \mu)$. In particular, if the action of $\Gamma$ is proper and all bounded $\mu$-harmonic functions on $X$ are constant, then $\Gamma$ is amenable.

The proof of Theorem G' consists of a translation of the proof of [LS, Theorem 3'] to the discrete case.

Proof of Theorem G" Let $m: L^{\infty}\left(\mathbb{N}_{0}\right) \rightarrow \mathbb{R}$ be an invariant mean for the Abelian semigroup $\mathbb{N}_{0}$, that is, $m$ is a functional with $m(1)=1, m(f) \geq 0$ if $f \geq 0$, and $m\left(f_{k}\right)=m(f)$ for all $f \in L^{\infty}\left(\mathbb{N}_{0}\right)$ and $k \in \mathbb{N}_{0}$, where $f_{k}(l)=f(k+l)$.

Let $x \in X$ and $f \in L^{\infty}(X)$. Then $f_{x}: \mathbb{N}_{0} \rightarrow \mathbb{R}, f_{x}(k)=\mu_{x}^{k}(f)$, belongs to $L^{\infty}\left(\mathbb{N}_{0}\right)$, and we set

$$
\hat{m}: L^{\infty}(X) \rightarrow L^{\infty}(X), \quad \hat{m}(f)(x)=m\left(f_{x}\right)
$$

Then $\|\hat{m}\| \leq\|m\|=1$ and $\hat{m}(f)=f$ if $f$ is $\mu$-harmonic. Moreover, $\hat{m}$ is $\Gamma$-invariant, since $\mu$ is $\Gamma$-invariant. Furthermore,

$$
\begin{aligned}
\hat{m}(\mu(f))(x)=m\left((\mu(f))_{x}\right) & =m\left(\left(k \mapsto \mu_{x}^{k+1}(f)\right)\right) \\
& =m\left(\left(f_{x}\right)_{1}\right)=m\left(f_{x}\right)=\hat{m}(f)(x)
\end{aligned}
$$

since $m$ is an invariant mean. Now

$$
\begin{aligned}
\mu(f)_{x}(k) & =\sum_{y, z \in X} \mu_{x}^{k}(y) \mu_{y}(z) f(z) \\
& =\sum_{y, z \in X} \mu_{x}(y) \mu_{y}^{k}(z) f(z) \\
& =\sum_{y \in X} \mu_{x}(y) f_{y}(k) .
\end{aligned}
$$

For an exhausting sequence $F_{1} \subseteq F_{2} \subseteq \ldots$ of finite subsets of $X$, we then obtain

$$
\left|\mu(f)_{x}(k)-\sum_{y \in F_{n}} \mu_{x}(y) f_{y}(k)\right| \leq \sum_{y \in X \backslash F_{n}} \mu_{x}(y)\left\|\mu^{k}(f)\right\|_{\infty} \leq \sum_{y \in X \backslash F_{n}} \mu_{x}(y)\|f\|_{\infty}
$$

for all $k \in \mathbb{N}_{0}$. Therefore

$$
\left\|\mu(f)_{x}-\sum_{y \in F_{n}} \mu_{x}(y) f_{y}\right\| \leq \sum_{y \in X \backslash F_{n}} \mu_{x}(y)\|f\|_{\infty} .
$$


Since

$$
\begin{aligned}
\left|\hat{m}(f)(x)-\sum_{y \in F_{n}} \mu_{x}(y) \hat{m}(f)(y)\right| & =\left|m\left(\mu(f)_{x}\right)-\sum_{y \in F_{n}} \mu_{x}(y) m\left(f_{y}\right)\right| \\
& =\left|m\left(\mu(f)_{x}\right)-m\left(\sum_{y \in F_{n}} \mu_{x}(y) f_{y}\right)\right| \\
& \leq\|m\|\left\|\mu(f)_{x}-\sum_{y \in F_{n}} \mu_{x}(y) f_{y}\right\| \\
& \leq\|m\| \sum_{y \in X \backslash F_{n}} \mu_{x}(y)\|f\|_{\infty},
\end{aligned}
$$

which tends to 0 as $n \rightarrow \infty$, we conclude that

$$
\hat{m}\left(\mu(f)_{x}\right)=\sum_{y \in X} \mu_{x}(f) \hat{m}(f)(y),
$$

that is, $\hat{m}(f)$ is a $\mu$-harmonic function on $X$. The first assertion follows.

If the action of $\Gamma$ is proper, then the isotropy groups $\Gamma_{x}$ of $\Gamma$ are finite. Fixing a set $R \subseteq X$ of representatives of the $\Gamma$-orbits in $X$, we define

$$
E: L^{\infty}(\Gamma) \rightarrow L^{\infty}(X), \quad E(f)(\gamma x)=\frac{1}{\left|\Gamma_{x}\right|} \sum_{\sigma \in \Gamma_{x}} f(\gamma \sigma),
$$

where $x \in R$. Then $\|E\| \leq 1$ and $E(1)=1$. Furthermore, $E$ is $\Gamma$-equivariant,

$$
\begin{aligned}
E\left(\tau^{*} f\right)(\gamma x) & =\frac{1}{\left|\Gamma_{x}\right|} \sum_{\sigma \in \Gamma_{x}}\left(\tau^{*} f\right)(\gamma \sigma) \\
& =\frac{1}{\left|\Gamma_{x}\right|} \sum_{\sigma \in \Gamma_{x}} f(\tau \gamma \sigma) \\
& =E(f)(\tau \gamma x)=\left(\tau^{*} E(f)\right)(\gamma x) .
\end{aligned}
$$

Hence $\hat{m}$ induces an invariant mean on $\Gamma$ if $\mathcal{H}^{\infty}(X, \mu)$ is trivial.

Remark 6.18. For the second assertion of Theorem G' we only need that the stabilizers of the $\Gamma$-action on $X$ are amenable. Then we would set $E(f)(\gamma x)=$ $m_{x}\left(\gamma^{*} f\right)$, where $x \in R$ and $m_{x}$ is an invariant mean for $\Gamma_{x}$. 


\section{REFERENCES}

[An] A. Ancona, Negatively curved manifolds, elliptic operators, and the Martin boundary. Ann. of Math. 125 (1987), no. 3, 495-536.

[AS] M. T. Anderson and R. Schoen, Positive harmonic functions on complete manifolds of negative curvature. Ann. of Math. 121 (1985), no. 3, 429-461.

[BBE] M. Babillot, P. Bougerol, and L. Elie, On the strong Liouville property for co-compact Riemannian covers. Rend. Sem. Mat. Fis. Milano 64 (1994), 77-84 (1996).

[BL1] W. Ballmann and F. Ledrappier, The Poisson boundary for rank one manifolds and their cocompact lattices. Forum Math. 6 (1994), no. 3, 301-313.

[BL2] W. Ballmann and F. Ledrappier, Discretization of positive harmonic functions on Riemannian manifolds and Martin boundary. Actes de la Table Ronde de Géométrie Différentielle (Luminy, 1992), 77-92, Sémin. Congr. 1, Soc. Math. France, Paris, 1996.

[BMP] W. Ballmann, H. Matthiesen, and P. Polymerakis, On the bottom of spectra under coverings. Math. Zeitschrift 288 (2018), 1029-1036.

[BE] P. Bougerol and L. Elie, Existence of positive harmonic functions on groups and on covering manifolds. Ann. Inst. H. Poincar Probab. Statist. 31 (1995), no. 1, 59-80.

[CY] S. Y. Cheng and S. T. Yau, Differential equations on Riemannian manifolds and their geometric applications. Comm. Pure Appl. Math. 28 (1975), no. 3, 333-354.

[CD] G. Choquet and J. Deny, Sur l'équation de convolution $\mu=\mu * \sigma$. C. R. Acad. Sci. Paris 250 (1960), 799-801.

[Ec] S. Echterhoff, On maximal prime ideals in certain group $C^{*}$-algebras and crossed product algebras. J. Operator Th. 23 (1990), 317-338.

[Ep] C. L. Epstein, Positive harmonic functions on abelian covers. J. Funct. Anal. 82 (1989), no. 2, 303-315.

[Er] A. Erschler, Liouville property for groups and manifolds. Invent. Math. 155 (2004), no. 1, 55-80.

[FS] D. Fischer-Colbrie and R. Schoen, The structure of complete stable minimal surfaces in 3-manifolds of nonnegative scalar curvature. Comm. Pure Appl. Math. 33 (1980), no. 2, 199-211.

[K1] V.A. Kaimanovich, Brownian motion and harmonic functions on covering manifolds. An entropy approach. Soviet Math. Dokl. 33 (1986), no. 3, 812-816.

[K2] V.A. Kaimanovich, Discretization of bounded harmonic functions on Riemannian manifolds and entropy. Potential theory (Nagoya, 1990), 213-223, de Gruyter, Berlin, 1992.

[KV] V.A. Kaimanovich and A. M. Vershik, Random walks on discrete groups: boundary and entropy. Ann. Probab. 11 (1983), no. 3, 457-490.

[Ki] Y. Kifer, Brownian motion and positive harmonic functions on complete manifolds of nonpositive curvature. From local times to global geometry, control and physics. (Coventry, 1984/85), 187-232, Pitman Res. Notes Math. Ser., 150, Longman Sci. Tech., Harlow, 1986.

[Li] V. Ya. Lin, Liouville coverings of complex spaces, and amenable groups. (Russian) Mat. Sb. (N.S.) 132(174) (1987), no. 2, 202-224; translation in Math. USSR-Sb. 60 (1988), no. 1, 197-216.

[LZ] V. Lin and M. Zaidenberg, Liouville and Carathéodory coverings in Riemannian and complex geometry. Voronezh Winter Mathematical Schools, 111-130, Amer. Math. Soc. Transl. Ser. 2, 184, Adv. Math. Sci., 37, Amer. Math. Soc., Providence, RI, 1998.

[LS] T. Lyons and D. Sullivan, Function theory, random paths and covering spaces. J. Differential Geom. 19 (1984), no. 2, 299-323.

[Ma] G. A. Margulis, Positive harmonic functions on nilpotent groups. Dokl. Akad. Nauk SSSR 166 1054-1057 (Russian); translated as Soviet Math. Dokl. 71966 241-244.

[P1] P. Polymerakis, Coverings preserving the bottom of the spectrum. MPI-Preprint 2019-3, https://arxiv.org/abs/1811.07844

[P2] P. Polymerakis, Positive harmonic functions on covering spaces. In preparation.

[Su] D. Sullivan, Related aspects of positivity in Riemannian geometry. J. Differential Geom. 25 (1987), no. 3, 327-351,

WB: Max Planck Institute for Mathematics, Vivatsgasse 7, 53111 Bonn.

E-mail address: hwbllmnn@mpim-bonn.mpg.de

PP: Max Planck Institute for Mathematics, Vivatsgasse 7, 53111 Bonn.

E-mail address: polymerp@mpim-bonn.mpg.de 